\title{
MAXIMAL OPERATORS FOR THE $p$-LAPLACIAN FAMILY
}

\author{
PABLO BLANC, JUAN P. PINASCO, AND JULIO D. ROSSI
}

\begin{abstract}
We prove existence and uniqueness of viscosity solutions for the following problem:

$$
\max \left\{-\Delta_{p_{1}} u(x),-\Delta_{p_{2}} u(x)\right\}=f(x)
$$

in a bounded smooth domain $\Omega \subset \mathbb{R}^{N}$ with $u=g$ on $\partial \Omega$. Here $-\Delta_{p} u=$ $(N+p)^{-1}|D u|^{2-p} \operatorname{div}\left(|D u|^{p-2} D u\right)$ is the 1-homogeneous $p$-Laplacian and we assume that $2 \leq p_{1}, p_{2} \leq \infty$. This equation appears naturally when one considers a tug-of-war game in which one of the players (the one who seeks to maximize the payoff) can choose at every step which are the parameters of the game that regulate the probability of playing a usual Tugof-War game (without noise) or to play at random. Moreover, the operator $\max \left\{-\Delta_{p_{1}} u(x),-\Delta_{p_{2}} u(x)\right\}$ provides a natural analogous with respect to $p$-Laplacians to the Pucci maximal operator for uniformly elliptic operators.

We provide two different proofs of existence and uniqueness for this problem. The first one is based in pure PDE methods (in the framework of viscosity solutions) while the second one is more connected to probability and uses game theory.
\end{abstract}

\section{INTRODUCTION}

In this paper our goal is to show existence and uniqueness of viscosity solutions to the Dirichlet problem for the maximal operator associated with the family of $p$-Laplacian operators, $-\Delta_{p} u=-\operatorname{div}\left(|\nabla u|^{p-2} \nabla u\right)$ with $2 \leq p \leq \infty$.

When one considers the family of uniformly elliptic second order operators of the form $-\operatorname{tr}\left(A D^{2} u\right)$ and look for maximal operators one finds the so-called Pucci maximal operator, $P_{\lambda, \Lambda}^{+}\left(D^{2} u\right)=\max _{A \in \mathcal{A}}-\operatorname{tr}\left(A D^{2} u\right)$, where $\mathcal{A}$ is the set of uniformly elliptic matrices with ellipticity constant between $\lambda$ and $\Lambda$. This maximal operator plays a crucial role in the regularity theory for uniformly elliptic second order operators and has the following properties, see [7]:

(1) (Monotonicity) If $\lambda_{1} \leq \lambda_{2} \leq \Lambda_{2} \leq \Lambda_{1}$ then $P_{\lambda_{2}, \Lambda_{2}}^{+}\left(D^{2} u\right) \leq P_{\lambda_{1}, \Lambda_{1}}^{+}\left(D^{2} u\right)$.

(2) (Positively homogeneous) If $\alpha \geq 0$, then $P_{\lambda, \Lambda}^{+}\left(\alpha D^{2} u\right)=\alpha P_{\lambda, \Lambda}^{+}\left(D^{2} u\right)$.

(3) (Subsolutions) If $u$ verifies $P_{\lambda, \Lambda}^{+}\left(D^{2} u\right) \leq 0$ in the viscosity sense, then $-\operatorname{tr}\left(A D^{2} u\right) \leq 0$ for every matrix $A$ with ellipticity constants $\lambda$ and $\Lambda$ (that is, a subsolution to the maximal operator is a subsolution for every elliptic operator in the class). Therefore, from the comparison principle we get that a solution to $P_{\lambda, \Lambda}^{+}\left(D^{2} u\right) \leq 0$ provides a lower bound for every solution of any elliptic operator in the class with the same boundary values.

Date: April 3, 2015

2010 Mathematics Subject Classification. 35J70, 49N70, 91A15, 91A24.

Key words and phrases. Dirichlet boundary conditions, Dynamic Programming Principle, $p$ Laplacian, Tug-of-War games. 
If we try to reproduce these properties for the family of $p$-Laplacians we are lead to consider the operator $\max _{p_{1} \leq p \leq p_{2}}-\Delta_{p} u(x)$. As we will show in this paper, this operator has similar properties to the ones that hold for the Pucci maximal operator, but with respect to the $p$-Laplacian family.

Hence, it is natural to consider the Dirichlet problem for the partial differential equation

$$
\max _{p_{1} \leq p \leq p_{2}}-\Delta_{p} u(x)=f(x)
$$

in a bounded smooth domain $\Omega \subset \mathbb{R}^{N}$ for $2 \leq p_{1}, p_{2} \leq \infty$. Here we have normalized the $p$-Laplacian and considered the operator

$$
\Delta_{p} u=\frac{\operatorname{div}\left(|\nabla u|^{p-2} \nabla u\right)}{(N+p)|\nabla u|^{p-2}},
$$

that is called the 1 -homogeneus $p$-laplacian. We will assume that $f \equiv 0$ or that $f$ is strictly positive or negative in $\Omega$. We will call solutions (along the whole paper we consider solutions in the viscosity sense, see [9]) to this problem with $f \equiv 0, u$, as $p_{1}$ - $p_{2}$-harmonic functions.

Note that, formally, the 1-homogeneus $p$-laplacian can be written as

$$
\Delta_{p} u=\frac{p-2}{N+p} \Delta_{\infty} u+\frac{1}{N+p} \Delta u
$$

where $\Delta u$ is the usual Laplacian and $\Delta_{\infty} u$ is the normalized $\infty$-Laplacian, that is,

$$
\Delta u=\sum_{i=1}^{N} u_{x_{i} x_{i}} \quad \text { and } \quad \Delta_{\infty} u=\frac{1}{|\nabla u|^{2}} \sum_{i, j=1}^{N} u_{x_{i}} u_{x_{i} x_{i}} u_{x_{j}} .
$$

Therefore, we can think about the 1-homogeneus $p$-laplacian as a convex combination of the laplacian divided by $N+2$ and the $\infty$-laplacian, in fact,

$$
\Delta_{p} u=\frac{p-2}{N+p} \Delta_{\infty} u+\frac{N+2}{N+p} \frac{\Delta u}{N+2}=\alpha \Delta_{\infty} u+\theta \Delta u
$$

with $\alpha=\frac{p-2}{N+p}$ and $\theta=\frac{1}{N+p}$ (we reserve $\beta$ for a different constant) for $2 \leq p<\infty$, and $\alpha=1$ and $\theta=0$ for $p=\infty$.

Since we are dealing with convex combinations, equation (1.1) becomes

$$
\max _{p_{1} \leq p \leq p_{2}}-\Delta_{p} u(x)=\max \left\{-\Delta_{p_{1}} u(x),-\Delta_{p_{2}} u(x)\right\}=f(x)
$$

with $2 \leq p_{1}, p_{2} \leq \infty$.

Our main result reads as follows:

Theorem 1.1. Assume that $\inf _{\Omega} f>0, \sup _{\Omega} f<0$ or $f \equiv 0$. Then, given $g$ a continuous function defined on $\partial \Omega$, there exists a unique viscosity solution $u \in C(\bar{\Omega})$ of (1.2) with $u=g$ in $\partial \Omega$.

Moreover, a comparison principle holds, if $u, v \in C(\bar{\Omega})$ are such that

$$
\max \left\{-\Delta_{p_{1}} u,-\Delta_{p_{2}} u\right\} \leq f \quad \max \left\{-\Delta_{p_{1}} v,-\Delta_{p_{2}} v\right\} \geq f
$$

in $\Omega$ and $v \geq u$ on $\partial \Omega$, then $v \geq u$ in $\Omega$. 
In addition, we have a Hopf's lemma: let $u$ be a supersolution to (1.2) and $x_{0} \in \partial \Omega$ be such that $u\left(x_{0}\right)>u(x)$ for all $x \in \Omega$, then we have

$$
\limsup _{t \rightarrow 0^{+}} \frac{u\left(x_{0}-t \nu\right)-u\left(x_{0}\right)}{t}<0 .
$$

where $\nu$ is exterior normal to $\partial \Omega$.

Remark 1.2. An analogous result holds for the equation $\min _{p_{1} \leq p \leq p_{2}}-\Delta_{p} u(x)=f$.

Remark 1.3. For the homogeneous case, $f \equiv 0$, we have that viscosity sub and supersolutions to the 1-homogeneus $p$-Laplacian, $-\frac{p-2}{N+p} \Delta_{\infty} u-\frac{1}{N+p} \Delta u=0$, coincide with viscosity sub and supersolutions to the usual ( $p-1$ homogeneous) $p$-Laplacian $-\operatorname{div}\left(|\nabla u|^{p-2} \nabla u\right)=0$, see [30].

Therefore, for $f \equiv 0$ we are providing existence and uniqueness of viscosity solutions to $\max _{p_{1} \leq p \leq p_{2}}-\Delta_{p} u(x)=0$, being $\Delta_{p} u$ the usual $p$-Laplacian that comes from calculus of variations.

Remark 1.4. This maximal operator for the $p$-Laplacian family has the following properties that are analogous to the ones described above for Pucci's operator:

(1) (Monotonicity) If $p_{1,1} \leq p_{2,1} \leq p_{2,2} \leq p_{1,2}$ then

$$
\max _{p_{2,1} \leq p \leq p_{2,2}}-\Delta_{p} u \leq \max _{p_{1,1} \leq p \leq p_{1,2}}-\Delta_{p} u .
$$

(2) (Positively homogeneous) If $\alpha \geq 0$, then

$$
\max _{p_{1} \leq p \leq p_{2}}-\Delta_{p}(\alpha u)=\alpha \max _{p_{1} \leq p \leq p_{2}}-\Delta_{p} u .
$$

(3) (Subsolutions) A viscosity solution $u$ to $\max _{p_{1} \leq p \leq p_{2}}-\Delta_{p} u(x) \leq 0$, is a viscosity solution to $-\Delta_{p} u(x) \leq 0$ for every $p_{1} \leq p \leq p_{2}$. Hence, from the comparison principle we get that a solution to $\max _{p_{1} \leq p \leq p_{2}}-\Delta_{p} u(x) \leq 0$, provides a lower bound for every solution of any elliptic operator in the class with the same boundary values.

We have two different approaches for this problem. The first one is based in PDE tools in the framework of viscosity solutions. The second one is related to probability theory (game theory) using the game that we describe below.

Let us introduce a game that we call unbalanced Tug-of-War game with noise. It is a two-player (Players I and II) zero-sum stochastic game. The game is played in a bounded open set $\Omega \subset \mathbb{R}^{N}$. Fix an $\varepsilon>0$. At the initial time, the players place a token at a point $x_{0} \in \Omega$ and Player I chooses a coin between two possible ones. They toss the chosen coin which is biased with probabilities $\alpha_{i}$ and $\beta_{i}, \alpha_{i}+\beta_{i}=1$ and $1 \geq \alpha_{i}, \beta_{i} \geq 0, i=1,2$. Now, they play the Tug-of-War with noise game described in [30] with probabilities $\alpha_{i}, \beta_{i}$. If they get heads (probability $\alpha_{i}$ ), they toss a fair coin (with equal probability of heads and tails) and the winner of the toss moves the game position to any $x_{1} \in B_{\varepsilon}\left(x_{0}\right)$ of his choice. On the other hand, if they get tails (probability $\beta_{i}$ ) the game state moves according to the uniform probability density to a random point $x_{1} \in B_{\varepsilon}\left(x_{0}\right)$. Once the game position leaves $\Omega$, let say at the $\tau$-th step, the game ends. The payoff is given by a running payoff function $f: \Omega \rightarrow \mathbb{R}$ and a final payoff function $g: \mathbb{R}^{N} \backslash \Omega \rightarrow \mathbb{R}$ (note that we only use the values of $g$ in a strip of width $\varepsilon$ around $\partial \Omega$ ). At the end Player II pays to Player I the amount given by the formula $g\left(x_{\tau}\right)+\varepsilon^{2} \sum_{n=0}^{\tau-1} f\left(x_{n}\right)$. Note that the positions of the game depend on the strategies adopted by Players I and II. From 
this procedure we get two extreme functions, $u_{\mathrm{I}}\left(x_{0}\right)$ (the value of the game for Player I) and $u_{\mathrm{II}}\left(x_{0}\right)$ (the value of the game for Player II), that are in a sense the best expected outcomes that each player may expect choosing a strategy when the game starts at $x_{0}$. When $u_{\mathrm{I}}\left(x_{0}\right)$ and $u_{\mathrm{II}}\left(x_{0}\right)$ coincide at every $x_{0} \in \Omega$ this function $u_{\varepsilon}:=u_{\mathrm{I}}=u_{\mathrm{II}}$ is called the value of the game.

Theorem 1.5. Assume that $f$ is a Lipschitz function with $\sup _{\Omega} f<0$ or $\inf _{\Omega} f>0$ or $f \equiv 0$. The unbalanced Tug-of-War game with noise with $\alpha_{1}>0$ or $\alpha_{2}>0$ when $f \equiv 0$ has a value and that value satisfies the Dynamic Programming Principle, given by:

$u_{\varepsilon}(x)=\varepsilon^{2} f(x)+\max _{i \in\{1,2\}}\left(\frac{\alpha_{i}}{2}\left\{\sup _{y \in B_{\varepsilon}(x)} u_{\varepsilon}(y)+\inf _{y \in B_{\varepsilon}(x)} u_{\varepsilon}(y)\right\}+\beta_{i} f_{B_{\varepsilon}(x)} u_{\varepsilon}(y) d y\right)$

for $x \in \Omega$, with $u_{\varepsilon}(x)=g(x)$ for $x \notin \Omega$.

Moreover, if $g$ is Lipschitz, then there exists a uniformly continuous function $u$ such that

$$
u_{\varepsilon} \rightarrow u \quad \text { uniformly in } \bar{\Omega} \text {. }
$$

This limit $u$ is a viscosity solution to

$$
\begin{cases}\max \left\{-\Delta_{p_{1}} u,-\Delta_{p_{2}} u\right\}=\bar{f} & \text { on } \Omega, \\ u=g & \text { on } \partial \Omega,\end{cases}
$$

where $\bar{f}=2 f$ and $p_{1}, p_{2}$ are given by

$$
\alpha_{i}=\frac{p_{i}-2}{p_{i}+N} \quad, \quad \beta_{i}=\frac{2+N}{p_{i}+N}, \quad i=1,2 .
$$

Remark 1.6. When $f$ is strictly positive or negative we have that the game ends almost surely. The same is true (regardless the strategies adopted by the players) when they play with some noise at every turn, that is, when the two $\beta_{i}$ are positive. This fact simplifies the arguments used in the proofs.

When one of the $\alpha_{i}$ is one (and therefore the corresponding $\beta_{i}$ is zero) the argument is more delicate, see Section 4.

Remark 1.7. Note that in the limit problem one only considers the values of $g$ on $\partial \Omega$ while in the game one needs $g$ to be defined in a bigger set. Given a Lipschitz function defined on $\partial \Omega$ we can just extend it to this larger set without affecting the Lipschitz constant. For simplicity but making an abuse of notation we also call such extension as $g$.

Remark 1.8. We also prove uniqueness of solutions to the DPP, see Section 4. That is, there exists a unique function verifying

$$
v(x)=\varepsilon^{2} f(x)+\max _{i \in\{1,2\}}\left(\frac{\alpha_{i}}{2}\left\{\sup _{y \in B_{\varepsilon}(x)} v(y)+\inf _{y \in B_{\varepsilon}(x)} v(y)\right\}+\beta_{i} f_{B_{\varepsilon}(x)} v(y) d y\right)
$$

for $x \in \Omega$, with $v(x)=g(x)$ for $x \notin \Omega$.

Remark 1.9. When Player II (recall that this player wants to minimize the expected outcome) has the choice of the probabilities $\alpha$ and $\beta$ we end up with a solution to

$$
\begin{cases}\min \left\{-\Delta_{p_{1}} u,-\Delta_{p_{2}} u\right\}=f & \text { on } \Omega \\ u=g & \text { on } \partial \Omega\end{cases}
$$


Let us end the introduction with some brief comments on related work. First, let us recall that Pucci operators are crucial in regularity theory for uniformly elliptic operators, due to their natural comparison with a non-divergence linear operator with measurable coefficients. We refer to [6], [7], [10], [35] and references therein.

On the other hand, concerning probabilistic ideas for PDEs, the fundamental works of Doob, Hunt, Kakutani, Kolmogorov and many others have shown the profound and powerful connection between the classical linear potential theory and the corresponding probability theory. The idea behind the classical interplay is that harmonic functions and martingales share a common origin in mean value properties. This approach turns out to be useful in the nonlinear theory as well, since $p$-harmonic functions verify an asymptotic mean value property, see, for example, [28], [13], [16], [21] and [22]. Concerning Tug-of-War games and PDEs the story begins with [33] and [34] and was extended in [3], [4], [5], [31], etc. Remark that for the $p$-Laplacian it was proved in [14], [15] the equivalence between viscosity and weak solutions. This probability approach was used to obtain regularity properties of solutions, we refer to [2], [24], [25] and [36]. We will study regularity for solutions to our maximal operators in a forthcoming paper.

The paper is organized as follows: In Section 2 we prove the comparison principle and then existence and uniqueness for our problem using Perron's method; in Section 3 we introduce a precise description of the game; in Section 4 we show that the game has a value and that this value is the solution to the Dynamic Programming Principle; finally, in Section 5 we collect some properties of the value function of the game and show that these values converge to the unique viscosity solution of our problem.

\section{EXISTENCE AND UNIQUENESS}

First, let us state the definition of a viscosity solution. We have to handle some technical difficulties as the 1 -homogeneous $\infty$-laplacian is not well defined when the gradient vanish. Observing that

$$
\Delta u=\operatorname{tr}\left(D^{2} u\right) \quad \text { and } \quad \Delta_{\infty} u=\frac{\nabla u}{|\nabla u|} D^{2} u \frac{\nabla u}{|\nabla u|},
$$

we can write $(1.2)$ as $F\left(\nabla u, D^{2} u\right)=f$ where

$$
F(v, X)=\max _{i \in\{1,2\}}\left\{-\alpha_{i} \frac{v}{|v|} X \frac{v}{|v|}-\theta_{i} \operatorname{tr}(X)\right\}
$$

Note that $F$ is degenerate elliptic, that is,

$$
F(v, X) \leq F(v, Y) \text { for } v \in \mathbb{R}^{N} \backslash\{0\} \text { and } X, Y \in S^{N} \text { provided } X \geq Y,
$$

as it is generally requested to work in the context of viscosity solutions.

This function $F: \mathbb{R}^{N} \times S^{N} \mapsto \mathbb{R}$ is not well defined at $v=0$ (here $S^{N}$ denotes the set of real symmetric $N \times N$ matrices). Therefore, we need to consider the lower semicontinous $F_{*}$ and upper semicontinous $F^{*}$ envelopes of $F$. This functions coincide with $F$ for $v \neq 0$ and for $v=0$ are given by

$$
F^{*}(0, X)=\max _{i \in\{1,2\}}\left\{-\alpha_{i} \lambda_{\min }(X)-\theta_{i} \operatorname{tr}(X)\right\}
$$


and

$$
F_{*}(0, X)=\max _{i \in\{1,2\}}\left\{-\alpha_{i} \lambda_{\max }(X)-\theta_{i} \operatorname{tr}(X)\right\}
$$

where $\lambda_{\min }(X)=\min \{\lambda: \lambda$ is an eigenvalue of $X\}$ and $\lambda_{\max }(X)=\max \{\lambda$ : $\lambda$ is an eigenvalue of $X\}$.

Now we are ready to give the definition for a viscosity solution to our equation.

Definition 2.1. For $2 \leq p_{1}, p_{2} \leq \infty$ consider the equation

$$
\max \left\{-\Delta_{p_{1}} u,-\Delta_{p_{2}} u\right\}=f
$$

in $\Omega$.

(1) A lower semi-continuous function $u$ is a viscosity supersolution if for every $\phi \in C^{2}$ such that $\phi$ touches $u$ at $x \in \Omega$ strictly from below, we have

$$
F^{*}\left(\nabla \phi(x), D^{2} \phi(x)\right) \geq f(x) .
$$

(2) An upper semi-continuous function $u$ is a subsolution if for every $\psi \in C^{2}$ such that $\psi$ touches $u$ at $x \in \Omega$ strictly from above, we have

$$
F_{*}\left(\nabla \psi(x), D^{2} \psi(x)\right) \leq f(x) .
$$

(3) Finally, $u$ is a viscosity solution if it is both a sub-and supersolution.

In the case $f \equiv 0$ comparison holds for our equation as a consequence of the main result of [17]. Remark that in [17] a different notion of viscosity solution is considered. We remark that when a function is a viscosity sub or super-solution in the sense of Definition 2.1 it is also that in the sense considered in [17]. Therefore we can use the comparison result established there once we check their hypotheses.

Proposition 2.2. Let $u \in U S C(\Omega)$ and $v \in L S C(\Omega)$ be, respectively, a viscosity subsolution and a viscosity supersolution of (1.2) with $f \equiv 0$. If $u \leq v$ on $\partial \Omega$, then $u \leq v$ in $\Omega$.

Proof. We just apply the main result in [17]. To this end we need to check some conditions (we refer to [17] for notations and details). First, let us show that $F$ is elliptic, in fact we have

$$
\begin{aligned}
F(v, X-\mu v \otimes v) & =\max _{i \in\{1,2\}}\left\{-\alpha_{i} \frac{v}{|v|}(X-\mu v \otimes v) \frac{v}{|v|}-\theta_{i} \operatorname{tr}(X-\mu v \otimes v)\right\} \\
& =\max _{i \in\{1,2\}}\left\{-\alpha_{i} \frac{v}{|v|} X \frac{v}{|v|}+\alpha_{i} \mu|v|^{2}-\theta_{i} \operatorname{tr}(X)+\theta_{i} \mu|v|^{2}\right\} \\
& =\max _{i \in\{1,2\}}\left\{-\alpha_{i} \frac{v}{|v|} X \frac{v}{|v|}-\theta_{i} \operatorname{tr}(X)+\theta_{i}\right\}+\mu|v|^{2} \\
& =F(v, X)+\mu|v|^{2} .
\end{aligned}
$$

Moreover, $F$ is invariant by rescaling in $v$ and 1-homogeneous in $X$.

So, we can take $\sigma_{0}(v)=|v|^{2}, \sigma_{1}(t)=t$ and $\rho \equiv 0$ (using the notation from [17]) that satisfy the conditions imposed in [17] to obtain the comparison result.

Now we deal with the case where $f$ is assumed to be nontrivial and does not change sign. In fact, we assume that $\inf f>0$ or $\sup f<0$. We follow a similar ideas to the ones in [23]. 
Lemma 2.3. If we have $u, v \in C(\bar{\Omega})$ such that

$$
\max \left\{-\Delta_{p_{1}} u,-\Delta_{p_{2}} u\right\} \leq f \quad \max \left\{-\Delta_{p_{1}} v,-\Delta_{p_{2}} v\right\} \geq g
$$

where $g>f$ and $v \geq u$ in $\partial \Omega$, then we have $v \geq u$ in $\Omega$.

Proof. By adding a constant if necessary we can assume that $u, v>0$. Arguing by contradiction we assume that

$$
\max _{\bar{\Omega}}(u-v)>0 \geq \max _{\partial \Omega}(u-v) .
$$

Now we double the variables and consider

$$
\sup _{x, y \in \Omega}\left\{u(x)-v(y)-\frac{j}{2}|x-y|^{2}\right\} \text {. }
$$

For large $j$ the supremum is attained at interior points $x_{j}, y_{j}$ such that $x_{j} \rightarrow \hat{x}$, $y_{j} \rightarrow \hat{x}$, where $\hat{x}$ is an interior point (that $\hat{x}$ cannot be on the boundary can be obtained as in [18]).

Now, we observe that there exists a constant $C$ such that $j\left|x_{j}-y_{j}\right| \leq C$. The theorem of sums implies that there are symmetric matrices $\mathbb{X}_{j}, \mathbb{Y}_{j}$, with $\mathbb{X}_{j} \leq \mathbb{Y}_{j}$ such that $\left(j\left|x_{j}-y_{j}\right|, \mathbb{X}_{j}\right) \in \overline{J^{2,+}}(u)\left(x_{j}\right)$ and $\left(j\left|x_{j}-y_{j}\right|, \mathbb{Y}_{j}\right) \in \overline{J^{2,-}}(v)\left(y_{j}\right)$, where $\overline{J^{2,+}}(u)\left(x_{j}\right)$ and $\overline{J^{2,-}}(v)\left(y_{j}\right)$ are the closures of the super and subjets of $u$ and $v$ respectively. Using the equations, assuming that $x_{j} \neq y_{j}$, we have

and

$$
\max _{i \in\{1,2\}}\left\{-\alpha_{i}\left\langle\mathbb{X}_{j} \frac{\left(x_{j}-y_{j}\right)}{\left|x_{j}-y_{j}\right|}, \frac{\left(x_{j}-y_{j}\right)}{\left|x_{j}-y_{j}\right|}\right\rangle-\theta_{i} \operatorname{tr}\left(\mathbb{X}_{j}\right)\right\} \leq f\left(y_{j}\right)
$$

$$
\max _{i \in\{1,2\}}\left\{-\alpha_{i}\left\langle\mathbb{Y}_{j} \frac{\left(x_{j}-y_{j}\right)}{\left|x_{j}-y_{j}\right|}, \frac{\left(x_{j}-y_{j}\right)}{\left|x_{j}-y_{j}\right|}\right\rangle-\theta_{i} \operatorname{tr}\left(\mathbb{Y}_{j}\right)\right\} \geq g\left(y_{j}\right)
$$

Now we observe that, since $\mathbb{X}_{j} \leq \mathbb{Y}_{j}$ we get

$$
-\operatorname{tr}\left(\mathbb{X}_{j}\right) \geq-\operatorname{tr}\left(\mathbb{Y}_{j}\right)
$$

and

Hence

$$
-\left\langle\mathbb{X}_{j} \frac{\left(x_{j}-y_{j}\right)}{\left|x_{j}-y_{j}\right|}, \frac{\left(x_{j}-y_{j}\right)}{\left|x_{j}-y_{j}\right|}\right\rangle \geq-\left\langle\mathbb{Y}_{j} \frac{\left(x_{j}-y_{j}\right)}{\left|x_{j}-y_{j}\right|}, \frac{\left(x_{j}-y_{j}\right)}{\left|x_{j}-y_{j}\right|}\right\rangle
$$

$$
\begin{aligned}
f\left(y_{j}\right) & \geq \max _{i \in\{1,2\}}\left\{-\alpha_{i}\left\langle\mathbb{X}_{j} \frac{\left(x_{j}-y_{j}\right)}{\left|x_{j}-y_{j}\right|}, \frac{\left(x_{j}-y_{j}\right)}{\left|x_{j}-y_{j}\right|}\right\rangle-\theta_{i} \operatorname{tr}\left(\mathbb{X}_{j}\right)\right\} \\
& \geq \max _{i \in\{1,2\}}\left\{-\alpha_{i}\left\langle\mathbb{Y}_{j} \frac{\left(x_{j}-y_{j}\right)}{\left|x_{j}-y_{j}\right|}, \frac{\left(x_{j}-y_{j}\right)}{\left|x_{j}-y_{j}\right|}\right\rangle-\theta_{i} \operatorname{tr}\left(\mathbb{Y}_{j}\right)\right\} \geq g\left(x_{j}\right) .
\end{aligned}
$$

This gives a contradiction passing to the limit as $j \rightarrow \infty$.

When $x_{j}=y_{j}$ we obtain

$$
\max _{i \in\{1,2\}}\left\{-\alpha_{i} \lambda_{\max }\left(\mathbb{Y}_{j}\right)-\theta_{i} \operatorname{tr}\left(\mathbb{Y}_{j}\right)\right\} \leq f\left(y_{j}\right)
$$

and

$$
\max _{i \in\{1,2\}}\left\{-\alpha_{i} \lambda_{\min }\left(\mathbb{X}_{j}\right)-\theta_{i} \operatorname{tr}\left(\mathbb{X}_{j}\right)\right\} \geq g\left(x_{j}\right)
$$

that also leads to a contradiction since $\lambda_{\max }\left(\mathbb{Y}_{j}\right) \geq \lambda_{\max }\left(\mathbb{X}_{j}\right) \geq \lambda_{\min }\left(\mathbb{X}_{j}\right)$.

Hence we have obtained that $u \leq v$, as we wanted to prove. 
Lemma 2.4. If $u, v \in C(\bar{\Omega})$ are such that

$$
\max \left\{-\Delta_{p_{1}} u,-\Delta_{p_{2}} u\right\} \leq f \quad \max \left\{-\Delta_{p_{1}} v,-\Delta_{p_{2}} v\right\} \geq f
$$

in $\Omega$ with $\inf _{\Omega} f>0$ and $v \geq u$ on $\partial \Omega$, then we have $v \geq u$ in $\Omega$.

Proof. By adding a constant if necessary we can assume that $u, v>0$. Lets consider $v_{\delta}=(1+\delta) v$

$$
\max \left\{-\Delta_{p_{1}} u,-\Delta_{p_{2}} u\right\} \leq f<(1+\delta) f \leq \max \left\{-\Delta_{p_{1}} v_{\delta},-\Delta_{p_{2}} v_{\delta}\right\}
$$

and $v_{\delta} \geq v \geq u$ in $\partial \Omega$. Then by the preceding lemma we conclude that and $v_{\delta} \geq u$ in $\Omega$ for all $\delta>0$. Making $\delta \rightarrow 0$, we get $v \geq u$ in $\Omega$ as we wanted to show.

Remark 2.5. The above lemma is also true when $\sup _{\Omega} f<0$. So, we have comparison for the cases $\inf _{\Omega} f>0, \sup _{\Omega} f<0$ and $f \equiv 0$. From this comparison result we get uniqueness of solutions.

Now we deal with the existence of solutions. In the proof of this result we are only using that the exterior ball condition holds for $\Omega$ when $p_{1}, p_{2} \leq N$.

Theorem 2.6. Assume that $\inf f>0, \sup f<0$ or $f \equiv 0$. Then, given $g$ a continuous function defined on $\partial \Omega$, there exists $u \in C(\bar{\Omega})$ a viscosity solution of (1.2) such that $u=g$ in $\partial \Omega$.

Proof. We consider the set

$$
\mathcal{A}=\left\{v \in C(\bar{\Omega}): \max \left\{-\Delta_{p_{1}} v,-\Delta_{p_{2}} v\right\} \geq f \text { in } \Omega \text { and } v \geq g \text { on } \partial \Omega\right\},
$$

where the inequality for the equation inside $\Omega$ is verified in the viscosity sense and the inequality on $\partial \Omega$ in the pointwise sense. The set $\mathcal{A}$ is not empty, since the function $K_{2}-K_{1}|x|^{2} \in \mathcal{A}$ for suitable $K_{1}, K_{2}$. We define

$$
u(x)=\inf _{v \in \mathcal{A}} v(x), \quad x \in \bar{\Omega} .
$$

This infimum is finite since, as comparison holds, we have $u(x) \geq-L_{2}+L_{1}|x|^{2}$ for all $u \in \mathcal{A}$ for large $L_{1}, L_{2}$. The function $u$, being the infimum of supersolutions, is a supersolution. We already know that $u$ is upper semi-continuous, as it the infimum of continuous functions. Let us see it is indeed a solution. Suppose not, then there exist $\phi \in C^{2}$ such that $\phi$ touches $u$ at $x_{0} \in \Omega$ strictly from above but

$$
\max \left\{-\Delta_{p_{1}} \phi\left(x_{0}\right),-\Delta_{p_{2}} \phi\left(x_{0}\right)\right\}>f\left(x_{0}\right) .
$$

Lets write

$$
\phi(x)=\phi\left(x_{0}\right)+\nabla \phi\left(x_{0}\right) \cdot\left(x-x_{0}\right)+\frac{1}{2}\left\langle D^{2} \phi\left(x_{0}\right)\left(x-x_{0}\right), x-x_{0}\right\rangle+o\left(\left|x-x_{0}\right|^{2}\right)
$$

We define $\hat{\phi}(x)=\phi(x)-\delta$ for a small positive number $\delta$. Then $\hat{\phi}<u$ in a small neighborhood of $x_{0}$, contained in the set $\left\{x: \max \left\{-\Delta_{p_{1}} \phi(x),-\Delta_{p_{2}} \phi(x)\right\}>f(x)\right\}$, but $\hat{\phi} \geq u$ outside this neighborhood, if we take $\delta$ small enough.

Now we can consider $v=\min \{\hat{\phi}, u\}$. Since $u$ is a viscosity supersolution in $\Omega$ and $\hat{\phi}$ also is a viscosity supersolution in the small neighborhood of $x_{0}$, it follows that $v$ is a viscosity supersolution. Moreover, on $\partial \Omega, v=u \geq g$. This implies $v \in \mathcal{A}$, but $v=\hat{\phi}<u$ near $x_{0}$, which is a contradiction with the definition of $u$ as the infimum in $\mathcal{A}$. 
Finally we want to prove that $u=g$ in $\partial \Omega$ and that boundary values are achieved with continuity. To this end, it is enough to construct a barrier for our operator. Let us consider $\phi$ a radial function, $\phi(x)=\psi(r)$ with $\psi^{\prime}(r)>0$. Then

$$
\Delta_{\infty} \phi=\psi^{\prime \prime} \quad \text { and } \quad \Delta \phi=\psi^{\prime \prime}+\frac{N-1}{r} \psi^{\prime}
$$

and we get

$$
\begin{aligned}
\max _{i \in\{1,2\}}\left\{-\Delta_{p_{i}} \phi\right\} & =\max _{i \in\{1,2\}}\left\{-\alpha_{i} \Delta_{\infty} \phi-\theta_{i} \Delta \phi\right\} \\
& =\max _{i \in\{1,2\}}\left\{-\alpha_{i} \psi^{\prime \prime}-\theta_{i}\left(\psi^{\prime \prime}+\frac{N-1}{r} \psi^{\prime}\right)\right\} \\
& =\max _{i \in\{1,2\}}\left\{-\frac{p_{i}-2}{N+p_{i}} \psi^{\prime \prime}-\frac{1}{N+p_{i}}\left(\psi^{\prime \prime}+\frac{N-1}{r} \psi^{\prime}\right)\right\} \\
& =\max _{i \in\{1,2\}}\left\{-\frac{p_{i}-1}{N+p_{i}} \psi^{\prime \prime}-\frac{1}{N+p_{i}} \frac{N-1}{r} \psi^{\prime}\right\}
\end{aligned}
$$

We want this last expression to be greater than a positive constant. To have a function of the form $\psi(r)=r^{\gamma}, \gamma>0$, that fulfills this we need

$$
\max _{i \in\{1,2\}}\left\{-\frac{p_{i}-1}{N+p_{i}} \gamma(\gamma-1)-\frac{N-1}{N+p_{i}} \gamma\right\} r^{\gamma-2} \geq c>0
$$

Hence we choose $\gamma$ according to

$$
0<\gamma<1-\frac{N-1}{p_{i}-1}
$$

We have that such $\gamma$ exists if $N<p_{1}$ or $N<p_{2}$.

If this is not the case, we can find (with similar computations) a barrier of the form $\psi(r)=-r^{\gamma}$ with $\gamma<0$. In this case, when $N \geq p_{1}, p_{2}$, we have a barrier if the exterior ball condition holds.

Now, we prove a version of the Hopf lemma for our equation. Note that since we deal with viscosity solutions the normal derivative may not exists in a classical sense.

Lemma 2.7. Let $\Omega \subset \mathbb{R}^{N}$ be a domain with the interior ball condition and $u$ subsolution to (1.2) whith $f \equiv 0$. Given $x_{0} \in \partial \Omega$ such that $u\left(x_{0}\right)>u(x)$ for all $x \in \Omega$, we have

$$
\limsup _{t \rightarrow 0^{+}} \frac{u\left(x_{0}-t \nu\right)-u\left(x_{0}\right)}{t}<0 .
$$

where $\nu$ is exterior normal to $\partial \Omega$.

Proof. As the interior ball condition holds, we can assume there exist a ball centered at 0 , contained in $\Omega$ that has $x_{0}$ in its boundary, that is, we have $B_{r}(0) \subset \Omega$ and $x_{0} \in$ $\partial B_{r}(0)$. Let us consider $\phi(x)=\frac{1}{|x|^{N-2}}-\frac{1}{r^{N-2}}$ if $N>2$ and $\phi(x)=-\ln |x|+\ln (r)$ for $N=2$. It easy to check that

$$
\Delta \phi=0, \quad \Delta_{\infty} \phi \geq 0, \quad \text { in } B_{r}(0) \backslash\{0\} .
$$

So we have

$$
\begin{aligned}
\max \left\{-\Delta_{p_{1}} \phi,-\Delta_{p_{2}} \phi\right\} & \leq 0 & & \text { in } B_{r}(0) \backslash\{0\} \\
\phi & \equiv 0 & & \text { on } \partial B_{r}(0)
\end{aligned}
$$


As $u\left(x_{0}\right)>u(x)$ for all $x \in \Omega$, in particular on $\partial B_{\frac{r}{2}}(0)$, then there exists $\varepsilon>0$ such that $u\left(x_{0}\right)-\varepsilon \phi \geq u$ on $\partial B_{\frac{r}{2}}(0)$. Therefore, by the comparison principle, we get $u\left(x_{0}\right)-\varepsilon \phi \geq u$ in $B_{r}(0) \backslash B_{\frac{r}{2}}(0)$ and the result follows.

\section{Unbalanced Tug-OF-WAR games With NOISE}

In this section we introduce the game that we call Unbalanced Tug-of-War game with noise. It is a two-player zero-sum stochastic game. The game is played over a bounded open set $\Omega \subset \mathbb{R}^{N}$. An $\varepsilon>0$ is given. Players I and II play as follows. At an initial time, they place a token at a point $x_{0} \in \Omega$ and Player I choose a coin between two possible ones (with different probabilities of getting heads for each coin), we think she chooses $i \in\{1,2\}$. Now they play the Tug-of-War with noise introduced in [30] starting with the chosen coin. They toss the chosen coin which is biased with probabilities $\alpha_{i}$ and $\beta_{i}, \alpha_{i}+\beta_{i}=1$ and $1 \geq \alpha_{i}, \beta_{i} \geq 0$. If they get heads (probability $\alpha_{i}$ ), they toss a fair coin (with the same probability for heads and tails) and the winner of the toss moves the game position to any $x_{1} \in B_{\varepsilon}\left(x_{0}\right)$ of his choice. On the other hand, if they get tails (probability $\beta_{i}$ ) the game state moves according to the uniform probability density to a random point $x_{1} \in B_{\varepsilon}\left(x_{0}\right)$. Then they continue playing from $x_{1}$. At each turn Player I may change the choice of the coin.

This procedure yields a sequence of game states $x_{0}, x_{1}, \ldots$ Once the game position leaves $\Omega$, let say at the $\tau$-th step, the game ends. At that time the token will be on the compact boundary strip around $\Omega$ of width $\varepsilon$ that we denote

$$
\Gamma_{\varepsilon}=\left\{x \in \mathbb{R}^{n} \backslash \Omega: \operatorname{dist}(x, \partial \Omega) \leq \varepsilon\right\}
$$

The payoff is given by a running payoff function $f: \Omega \rightarrow \mathbb{R}$ and a final payoff function $g: \Gamma_{\varepsilon} \rightarrow \mathbb{R}$. At the end Player II pays Player I the amount given by a $g\left(x_{\tau}\right)+\varepsilon^{2} \sum_{n=0}^{\tau-1} f\left(x_{n}\right)$, that is, Player I have earned $g\left(x_{\tau}\right)+\varepsilon^{2} \sum_{n=0}^{\tau-1} f\left(x_{n}\right)$ while Player II have earned $-g\left(x_{\tau}\right)-\varepsilon^{2} \sum_{n=0}^{\tau-1} f\left(x_{n}\right)$. We can think that when the token leaves $x_{i}$ Player II pays Player I $\varepsilon^{2} f\left(x_{i}\right)$ and $g\left(x_{\tau}\right)$ when the game ends.

A strategy $S_{\mathrm{I}}$ for Player I is a pair of collections of measurable mappings $S_{\mathrm{I}}=$ $\left(\left\{\gamma^{k}\right\}_{k=0}^{\infty},\left\{S_{\mathrm{I}}^{k}\right\}_{k=0}^{\infty}\right)$, such that, given a partial history $\left(x_{0}, x_{1}, \ldots, x_{k}\right)$, Player I choose coin 1 with probability

$$
\gamma^{k}\left(x_{0}, x_{1}, \ldots, x_{k}\right)=\gamma \in[0,1]
$$

and the next game position is

$$
S_{\mathrm{I}}^{k}\left(x_{0}, x_{1}, \ldots, x_{k}\right)=x_{k+1} \in B_{\varepsilon}\left(x_{k}\right)
$$

if Player I wins the toss. Similarly Player II plays according to a strategy $S_{\mathrm{II}}=$ $\left\{S_{\mathrm{II}}^{k}\right\}_{k=0}^{\infty}$. Then, the next game position $x_{k+1} \in B_{\varepsilon}\left(x_{k}\right)$, given a partial history $\left(x_{0}, x_{1}, \ldots, x_{k}\right)$, is distributed according to the probability

$$
\begin{aligned}
& \pi_{S_{\mathrm{I}}, S_{\mathrm{II}}}\left(x_{0}, x_{1}, \ldots, x_{k}, A\right) \\
& \quad=\frac{\beta\left|A \cap B_{\varepsilon}\left(x_{k}\right)\right|}{\left|B_{\varepsilon}\left(x_{k}\right)\right|}+\frac{\alpha}{2} \delta_{S_{\mathrm{I}}^{k}\left(x_{0}, x_{1} \ldots, x_{k}\right)}(A)+\frac{\alpha}{2} \delta_{S_{\mathrm{II}}^{k}\left(x_{0}, x_{1}, \ldots, x_{k}\right)}(A),
\end{aligned}
$$

where $\gamma=\gamma^{k}\left(x_{0}, x_{1} \ldots, x_{k}\right), \alpha=\alpha_{1} \gamma+\alpha_{2}(1-\gamma), \beta=\beta_{1} \gamma+\beta_{2}(1-\gamma)$ and $A$ is any measurable set (note that $\alpha$ and $\beta$ depend on $S_{\mathrm{I}}$ and $\left(x_{0}, x_{1}, \ldots, x_{k}\right)$, we do not make this explicit to avoid overloading the notation). From now on, we shall omit $k$ and simply denote the strategies by $\gamma, S_{\mathrm{I}}$ and $S_{\mathrm{II}}$. 
Let $\Omega_{\varepsilon}=\Omega \cup \Gamma_{\varepsilon} \subset \mathbb{R}^{n}$ be equipped with the natural topology, and the $\sigma$-algebra $\mathcal{B}$ of the Lebesgue measurable sets. The space of all game sequences

$$
H^{\infty}=\left\{x_{0}\right\} \times \Omega_{\varepsilon} \times \Omega_{\varepsilon} \times \ldots
$$

is a product space endowed with the product topology.

Let $\left\{\mathcal{F}_{k}\right\}_{k=0}^{\infty}$ denote the filtration of $\sigma$-algebras, $\mathcal{F}_{0} \subset \mathcal{F}_{1} \subset \ldots$ defined as follows: $\mathcal{F}_{k}$ is the product $\sigma$-algebra generated by cylinder sets of the form $\left\{x_{0}\right\} \times A_{1} \times$ $\ldots \times A_{k} \times \Omega_{\varepsilon} \times \Omega_{\varepsilon} \ldots$ with $A_{i} \in \mathcal{B}$. For

$$
\omega=\left(x_{0}, \omega_{1}, \ldots\right) \in H^{\infty},
$$

we define the coordinate processes

$$
X_{k}(\omega)=\omega_{k}, \quad X_{k}: H^{\infty} \rightarrow \mathbb{R}^{n}, k=0,1, \ldots
$$

so that $X_{k}$ is an $\mathcal{F}_{k}$-measurable random variable. Moreover, $\mathcal{F}_{\infty}=\sigma\left(\bigcup \mathcal{F}_{k}\right)$ is the smallest $\sigma$-algebra so that all $X_{k}$ are $\mathcal{F}_{\infty}$-measurable. To denote the time when the game state reaches $\Gamma_{\varepsilon}$, we define a random variable

$$
\tau(\omega)=\inf \left\{k: X_{k}(\omega) \in \Gamma_{\varepsilon}, k=0,1, \ldots\right\},
$$

which is a stopping time relative to the filtration $\left\{\mathcal{F}_{k}\right\}_{k=0}^{\infty}$.

A starting point $x_{0}$ and the strategies $S_{\mathrm{I}}$ and $S_{\mathrm{II}}$ define (by Kolmogorov's extension theorem) a unique probability measure $\mathbb{P}_{S_{I}, S_{\text {II }}}^{x_{0}}$ in $H^{\infty}$ relative to the $\sigma$-algebra $\mathcal{F}^{\infty}$. We denote by $\mathbb{E}_{S_{\mathrm{I}}, S_{\mathrm{II}}}^{x_{0}}$ the corresponding expectation.

Then, if $S_{\mathrm{I}}$ and $S_{\mathrm{II}}$ denote the strategies adopted by Player I and II respectively, we define the expected payoff for Player I as

$$
V_{x_{0}, \mathrm{I}}\left(S_{\mathrm{I}}, S_{\mathrm{II}}\right)= \begin{cases}\mathbb{E}_{S_{\mathrm{I}}, S_{\mathrm{II}}}^{x_{0}}\left[g\left(X_{\tau}\right)+\varepsilon^{2} \sum_{n=1}^{\tau-1} f\left(x_{n}\right)\right] & \text { if the game ends a.s. } \\ -\infty & \text { otherwise, }\end{cases}
$$

and the the expected payoff for Player II as

$$
V_{x_{0}, \mathrm{II}}\left(S_{\mathrm{I}}, S_{\mathrm{II}}\right)= \begin{cases}\mathbb{E}_{S_{\mathrm{I}}, S_{\mathrm{II}}}^{x_{0}}\left[g\left(X_{\tau}\right)+\varepsilon^{2} \sum_{n=1}^{\tau-1} f\left(x_{n}\right)\right] & \text { if the game ends a.s. } \\ +\infty & \text { otherwise. }\end{cases}
$$

Note that we penalize both players when the games doesn't end a.s.

The value of the game for Player $I$ is given by

$$
u_{\mathrm{I}}\left(x_{0}\right)=\sup _{S_{\mathrm{I}}} \inf _{S_{\mathrm{II}}} V_{x_{0}, \mathrm{I}}\left(S_{\mathrm{I}}, S_{\mathrm{II}}\right)
$$

while the value of the game for Player II is given by

$$
u_{\mathrm{II}}\left(x_{0}\right)=\inf _{S_{\mathrm{II}}} \sup _{S_{\mathrm{I}}} V_{x_{0}, \mathrm{II}}\left(S_{\mathrm{I}}, S_{\mathrm{II}}\right) .
$$

When $u_{\mathrm{I}}=u_{\mathrm{II}}$ we say the game has a value $u:=u_{\mathrm{I}}=u_{\mathrm{II}}$. The values $u_{\mathrm{I}}\left(x_{0}\right)$ and $u_{\mathrm{II}}\left(x_{0}\right)$ are in a sense the best outcomes each player can expect when the game starts at $x_{0}$. For the measurability of the value functions we refer to [26] and [27]. 
A comment. It seems natural to consider a more general protocol to determine $\alpha$ in a prescribed closed set. It is clear that there are only two possible scenarios: At each turn Player I wants to maximize the value of $\alpha$ and Player II wants to minimize it, or the converse. An expected value for $\alpha$ is obtained in each case assuming each player plays optimal. Depending on the value of $\alpha$ in each case, we are considering a game equivalent to the one that we described previously or another one where Player II gets the choice of the first coin, for certain values of $\alpha_{i}$.

\section{The game value function and the Dynamic Programming Principle}

In this section, we prove that the game has a value, that is, $u_{\mathrm{I}}=u_{\mathrm{II}}$ and that this value function satisfies the Dynamic Programming Principle (DPP) given by:

$$
\begin{aligned}
& u(x)=\varepsilon^{2} f(x)+\max _{i \in\{1,2\}}\left(\frac{\alpha_{i}}{2}\left\{\sup _{B_{\varepsilon}(x)} u+\inf _{B_{\varepsilon}(x)} u\right\}+\beta_{i} f_{B_{\varepsilon}(x)} u(y) d y\right), \quad x \in \Omega, \\
& u(x)=g(x), \quad x \in \Gamma_{\varepsilon} .
\end{aligned}
$$

Let see intuitively why this holds. At each step we have that Player I chooses $i \in\{1,2\}$ and then we have three possibilities:

- With probability $\frac{\alpha_{i}}{2}$, Player I moves the token, she will try to maximize the expected outcome.

- With probability $\frac{\alpha_{i}}{2}$, Player II moves the token, he will try minimize the expected outcome.

- With probability $\beta_{i}$, the token moves at random.

Since Player I chooses $i$ trying to maximize the expected outcome we obtain a $\max _{i \in\{1,2\}}$ in the DPP. Finally, the expected payoff at $x$ is given by $\varepsilon^{2} f(x)$ plus the expected payoff for the rest of the game.

Similar results are proved in [1], [20], [25], [29], [33] and [36]. Note that when $\alpha_{1}=\alpha_{2}$ (and hence $\beta_{1}=\beta_{2}$ ) player I has no choice to make and we recover known results for Tug-of-War games (with or without noise), see [33] and [30]. We follow [36] where the idea is to prove the existence of a function satisfying the DPP and then that this function gives the game value. For the existence of a solution to the DPP we borrow some ideas from [1], and for the uniqueness of such a solution and the existence of the value of the game we use martingales as in [29]. Although we will have two different cases. One where the noise assures us that the game end almost surely independently of the strategies adopted by the players or where the strictly positivity (or negativity) of $f$ helps us in this sense. And another one where we have to handle the problem of getting strategies for the players to play almost optimal and to make sure that the game ends almost surely.

In what follows $\Omega \subset \mathbb{R}^{N}$ is a bounded open set, $\varepsilon>0, g: \Gamma_{\varepsilon} \rightarrow \mathbb{R}$ and $f: \Omega \rightarrow \mathbb{R}$ bounded Borel functions such that $f \equiv 0, \inf _{\Omega} f>0$ or $\sup _{\Omega} f<0$.

Definition 4.1. A function $u$ is sub- $p_{1}-p_{2}$-harmonious if

$$
\begin{aligned}
& u(x) \leq \varepsilon^{2} f(x)+\max _{i \in\{1,2\}}\left(\frac{\alpha_{i}}{2}\left\{\sup _{B_{\varepsilon}(x)} u+\inf _{B_{\varepsilon}(x)} u\right\}+\beta_{i} f_{B_{\varepsilon}(x)} u(y) d y\right), \quad x \in \Omega, \\
& u(x) \leq g(x), \quad x \in \Gamma_{\varepsilon}
\end{aligned}
$$


Analogously, a function $u$ is super- $p_{1}-p_{2}$-harmonious if

$$
\begin{aligned}
& u(x) \geq \varepsilon^{2} f(x)+\max _{i \in\{1,2\}}\left(\frac{\alpha_{i}}{2}\left\{\sup _{B_{\varepsilon}(x)} u+\inf _{B_{\varepsilon}(x)} u\right\}+\beta_{i} f_{B_{\varepsilon}(x)} u(y) d y\right), \quad x \in \Omega, \\
& u(x) \leq g(x), \quad x \in \Gamma_{\varepsilon}
\end{aligned}
$$

Finally, $u$ is $p_{1}-p_{2}$-harmonious if it is both sub-and super-p $p_{1}-p_{2}$-harmonious (i.e. the equality holds).

Here $\alpha_{i}$ and $\beta_{i}$ are given by

$$
\alpha_{i}=\frac{p_{i}-2}{p_{i}+N} \quad \text { and } \quad \beta_{i}=\frac{N+2}{p_{i}+N} \quad i=1,2 .
$$

Our next task is to prove uniform bounds for these functions.

Lemma 4.2. Sub- $p_{1}-p_{2}$-harmonious functions are uniformly bounded from above.

Proof. Given $u$ a sub- $p_{1}-p_{2}$-subharmonious function, we have that

$$
u(x) \leq \varepsilon^{2} f(x)+\max _{i \in\{1,2\}}\left(\frac{\alpha_{i}}{2}\left\{\sup _{B_{\varepsilon}(x)} u+\inf _{B_{\varepsilon}(x)} u\right\}+\beta_{i} f_{B_{\varepsilon}(x)} u(y) d y\right)
$$

Given $x \in \Omega$ and $t \in \mathbb{R}$ such that $x_{n}<t \frac{\varepsilon}{2}+\frac{\varepsilon}{2}$ we have

$$
\left\{y \in B_{\varepsilon}(x): y_{n}<x_{n}-\frac{\varepsilon}{2}\right\} \subset\left\{z \in \mathbb{R}^{n}: z_{n}<t \frac{\varepsilon}{2}\right\} .
$$

We define

$$
D=\frac{\left|\left\{y \in B_{\varepsilon}: y_{n}<-\frac{\varepsilon}{2}\right\}\right|}{\left|B_{\varepsilon}\right|}=\frac{\left|\left\{y \in B_{1}: y_{n}<-\frac{1}{2}\right\}\right|}{\left|B_{1}\right|} \quad \text { and } \quad C=1-D .
$$

Considering this partition we have

$$
\begin{aligned}
& u(x) \leq \varepsilon^{2} \sup _{\Omega} f+\max _{i \in\{1,2\}}\left(\frac { \alpha _ { i } } { 2 } \left\{\sup _{\left.\Omega_{\varepsilon} u+\sup _{\Omega_{\varepsilon} \cap\left\{z_{n}<t \frac{\varepsilon}{2}\right\}} u\right\}}\right.\right. \\
&\left.+\beta_{i}\left\{C \sup _{\Omega_{\varepsilon}} u+D \sup _{\Omega_{\varepsilon} \cap\left\{z_{n}<t \frac{\varepsilon}{2}\right\}} u\right\}\right) \\
&=\varepsilon^{2} \sup _{\Omega} f+\max _{i \in\{1,2\}}\left(\left\{\frac{\alpha_{i}}{2}+\beta_{i} C\right\} \sup _{\Omega_{\varepsilon}} u+\left\{\frac{\alpha_{i}}{2}+\beta_{i} D\right\} \sup _{\Omega_{\varepsilon} \cap\left\{z_{n}<t \frac{\varepsilon}{2}\right\}} u\right) \\
&=\varepsilon^{2} \sup _{\Omega} f+\max _{i \in\{1,2\}}\left\{\frac{\alpha_{i}}{2}+\beta_{i} C\right\} \sup _{\Omega_{\varepsilon} u+\min _{i \in\{1,2\}}\left\{\frac{\alpha_{i}}{2}+\beta_{i} D\right\}} \sup _{\Omega_{\varepsilon} \cap\left\{z_{n}<t \frac{\varepsilon}{2}\right\}} u \\
&=\varepsilon^{2} \sup _{\Omega} f+K \sup _{\Omega_{\varepsilon}} u+(1-K) \sup _{\Omega_{\varepsilon} \cap\left\{z_{n}<t \frac{\varepsilon}{2}\right\}} u,
\end{aligned}
$$

where $K=\max _{i \in\{1,2\}}\left\{\frac{\alpha_{i}}{2}+\beta_{i} C\right\}$. We conclude that

$$
\sup _{\Omega_{\varepsilon} \cap\left\{z_{n}<(t+1) \frac{\varepsilon}{2}\right\}} u_{k} \leq \varepsilon^{2} \sup _{\Omega} f+K \sup _{\Omega_{\varepsilon}} u_{k}+(1-K) \sup _{\Omega_{\varepsilon} \cap\left\{z_{n}<t \frac{\varepsilon}{2}\right\}} u_{k} .
$$

Then, inductively, we get

$$
\sup _{\Omega_{\varepsilon} \cap\left\{z_{n}<(t+n) \frac{\varepsilon}{2}\right\}} u \leq\left(\varepsilon^{2} \sup _{\Omega} f+K \sup _{\Omega_{\varepsilon}} u\right) \sum_{i=0}^{n-1}(1-K)^{i}+(1-K)^{n} \sup _{\Omega_{\varepsilon} \cap\left\{z_{n}<t \frac{\varepsilon}{2}\right\}} u .
$$


Now we apply the formula for $t=0$ and $n$ such that $\frac{n \varepsilon}{2}>R$, we get

$$
\begin{aligned}
\sup _{\Omega_{\varepsilon}} u & \leq\left(\varepsilon^{2} \sup _{\Omega} f+K \sup _{\Omega_{\varepsilon}} u\right) \sum_{i=0}^{n-1}(1-K)^{i}+(1-K)^{n} \sup _{\Gamma_{\varepsilon}} g \\
& =\left(\varepsilon^{2} \sup _{\Omega} f+K \sup _{\Omega_{\varepsilon}} u\right) \frac{1-(1-K)^{n}}{1-(1-K)}+(1-K)^{n} \sup _{\Gamma_{\varepsilon}} g \\
& =\frac{1-(1-K)^{n}}{K} \varepsilon^{2} \sup _{\Omega} f+\left(1-(1-K)^{n}\right) \sup _{\Omega_{\varepsilon}} u+(1-K)^{n} \sup _{\Gamma_{\varepsilon}} F .
\end{aligned}
$$

Hence, we obtain

$$
(1-K)^{n} \sup _{\Omega_{\varepsilon}} u \leq \frac{1-(1-K)^{n}}{K} \varepsilon^{2} \sup _{\Omega} f+(1-K)^{n} \sup _{\Gamma_{\varepsilon}} g,
$$

that gives the desired upper bound,

$$
\sup _{\Omega_{\varepsilon}} u \leq \frac{1-(1-K)^{n}}{K(1-K)^{n}} \varepsilon^{2} \sup _{\Omega} f+\sup _{\Gamma_{\varepsilon}} g .
$$

Analogously, there holds that super- $p_{1}-p_{2}$-harmonious functions are uniformly bounded from below.

Now with this results we can show that there exists a $p_{1}-p_{2}$-harmonious function as in [19] applying Perron's Method. Remark that when $f$ and $g$ are bounded we can easily obtain the existence of sub- $p_{1}-p_{2}$-harmonious and super- $p_{1}$ - $p_{2}$-harmonious functions.

We prefer a constructive argument (since we will use again this construction in what follows). Let $u_{k}: \Omega_{\varepsilon} \rightarrow \mathbb{R}$ be a sequence of functions such that $u_{k}=g$ on $\Gamma_{\varepsilon}$ for all $k \in \mathbb{N}, u_{0}$ is sub- $p_{1}-p_{2}$-harmonious and

$$
u_{k+1}(x)=\varepsilon^{2} f(x)+\max _{i \in\{1,2\}}\left(\frac{\alpha_{i}}{2}\left\{\sup _{B_{\varepsilon}(x)} u_{k}+\inf _{B_{\varepsilon}(x)} u_{k}\right\}+\beta_{i} f_{B_{\varepsilon}(x)} u_{k}(y) d y\right)
$$

for $x \in \Omega$.

Now, our main task is to show that this sequence converges uniformly. To this end, let us prove an auxiliary lemma where we borrow some ideas from [1].

Lemma 4.3. Let $x \in \Omega, n \in \mathbb{N}$ and fix $\lambda_{i}$ for $i=1, \ldots, 4$ such that

$$
\begin{aligned}
u_{n+1}(x)-u_{n}(x) & \geq \lambda_{1}, \\
\left\|u_{n}-u_{n-1}\right\|_{\infty} & \leq \lambda_{2}, \\
f_{B_{\varepsilon}(x)} u_{n}-u_{n-1} & \leq \lambda_{3},
\end{aligned}
$$

$\lambda_{3}<\lambda_{1}$ and $\lambda_{4}>0$. Then, for $\alpha:=\max \left\{\alpha_{1}, \alpha_{2}\right\}>0$, there exists $y \in B_{\varepsilon}(x)$ such that

$$
u_{n}(x), u_{n}(y) \geq u_{n-1}(y)+\frac{2 \lambda_{1}}{\alpha}-\lambda_{2}-\frac{2(1-\alpha) \lambda_{3}}{\alpha}-\lambda_{4}
$$


Proof. Given $u_{n+1}(x)-u_{n}(x) \geq \lambda_{1}$, by the recursive definition, we have

$$
\begin{gathered}
\varepsilon^{2} f(x)+\max _{i \in\{1,2\}}\left(\frac{\alpha_{i}}{2}\left\{\sup _{B_{\varepsilon}(x)} u_{n}+\inf _{B_{\varepsilon}(x)} u_{n}\right\}+\beta_{i} f_{B_{\varepsilon}(x)} u_{n}(y) d y\right) \\
-\varepsilon^{2} f(x)-\max _{i \in\{1,2\}}\left(\frac{\alpha_{i}}{2}\left\{\sup _{B_{\varepsilon}(x)} u_{n-1}+\inf _{B_{\varepsilon}(x)} u_{n-1}\right\}+\beta_{i} f_{B_{\varepsilon}(x)} u_{n-1}(y) d y\right) \\
\geq \lambda_{1} .
\end{gathered}
$$

Since $\max \{a, b\}-\max \{c, d\} \leq \max \{a-c, b-d\}$, we get

$$
\begin{array}{r}
\max _{i \in\{1,2\}}\left(\frac{\alpha_{i}}{2}\left\{\sup _{B_{\varepsilon}(x)} u_{n}+\inf _{B_{\varepsilon}(x)} u_{n}-\sup _{B_{\varepsilon}(x)} u_{n-1}-\inf _{B_{\varepsilon}(x)} u_{n-1}\right\}+\right. \\
\left.\quad+\beta_{i} f_{B_{\varepsilon}(x)} u_{n}(y)-u_{n-1}(y) d y\right) \geq \lambda_{1} .
\end{array}
$$

Using that $f_{B_{\varepsilon}(x)} u_{n}-u_{n-1} \leq \lambda_{3}$ we get

$$
\max _{i \in\{1,2\}}\left(\frac{\alpha_{i}}{2}\left\{\sup _{B_{\varepsilon}(x)} u_{n}+\inf _{B_{\varepsilon}(x)} u_{n}-\sup _{B_{\varepsilon}(x)} u_{n-1}-\inf _{B_{\varepsilon}(x)} u_{n-1}\right\}+\beta_{i} \lambda_{3}\right) \geq \lambda_{1} .
$$

Now $\lambda_{3}<\lambda_{1}$ implies

$$
\frac{\alpha}{2}\left\{\sup _{B_{\varepsilon}(x)} u_{n}+\inf _{B_{\varepsilon}(x)} u_{n}-\sup _{B_{\varepsilon}(x)} u_{n-1}-\inf _{B_{\varepsilon}(x)} u_{n-1}\right\}+(1-\alpha) \lambda_{3} \geq \lambda_{1} .
$$

We bound the difference between the suprema using $\left\|u_{n}-u_{n-1}\right\|_{\infty} \leq \lambda_{2}$ and we obtain

$$
\frac{\alpha}{2}\left\{\inf _{B_{\varepsilon}(x)} u_{n}-\inf _{B_{\varepsilon}(x)} u_{n-1}\right\}+\frac{\alpha \lambda_{2}}{2}+(1-\alpha) \lambda_{3} \geq \lambda_{1},
$$

that is,

$$
\inf _{B_{\varepsilon}(x)} u_{n} \geq \inf _{B_{\varepsilon}(x)} u_{n-1}+\frac{2 \lambda_{1}}{\alpha}-\lambda_{2}-\frac{2(1-\alpha) \lambda_{3}}{\alpha} .
$$

Finally we can choose $y \in B_{\varepsilon}(x)$ such that

$$
u_{n-1}(y) \leq \inf _{B_{\varepsilon}(x)} u_{n-1}+\lambda_{4}
$$

which gives the desired inequality.

Now we are ready to prove the uniform convergence and, therefore, the existence of a $p_{1}$ - $p_{2}$-harmonious function.

Lemma 4.4. The sequence $u_{k}$ converges uniformly and the limit is a solution to the DPP.

Proof. Since $u_{0}$ is sub- $p_{1}-p_{2}$-harmonious we have $u_{1} \geq u_{0}$. In addition, if $u_{k} \geq u_{k-1}$, by the recursive definition, we have $u_{k+1} \geq u_{k}$. Then, by induction, we obtain that the sequence of functions is an increasing sequence. Replacing $u_{k} \leq u_{k+1}$ in the recursive definition we can see that $u_{k}$ is a sub- $p_{1}-p_{2}$-harmonious function for all $k$. This gives us a uniform bound for $u_{k}$ (independent of $k$ ). Hence, $u_{k}$ converge pointwise to a bounded Borel function $u$.

In the case $\alpha_{1}=\alpha_{2}=0$ we can pass to the limit on the recursion because of Fatou's Lemma. Hence we assume $\alpha:=\max \left\{\alpha_{1}, \alpha_{2}\right\}>0$. 
Now we show that the convergence is uniform. Suppose not. Observe that if $\left\|u_{n+1}-u_{n}\right\|_{\infty} \rightarrow 0$ we can extract a uniformly Cauchy subsequence, thus this subsequence converges uniformly to a limit $u$. This implies that $u_{k}$ converge uniformly to $u$, because of the monotonicity. By the recursive definition we have $\left\|u_{n+1}-u_{n}\right\|_{\infty} \geq\left\|u_{n}-u_{n-1}\right\|_{\infty} \geq 0$. Then, as we are assuming the convergence is not uniform, we have

for some $M>0$.

$$
\left\|u_{n+1}-u_{n}\right\|_{\infty} \rightarrow M
$$

Let us observe that by Fatou's Lemma it follows that

$$
\lim _{n \rightarrow \infty} \int_{\Omega} u(y)-u_{n}(y) d y=0
$$

so we can bound $f_{B_{\varepsilon}(x)} u_{n+1}-u_{n}$ uniformly on $x$.

Given $\delta>0$, let $n_{0} \in \mathbb{N}$ such that

$$
\left\|u_{n+1}-u_{n}\right\|_{\infty} \leq M+\delta \text { and } f_{B_{\varepsilon}(x)} u_{n+1}-u_{n}<\delta
$$

for all $x \in \Omega$. We fix $k \geq 0$. Let $x_{0} \in \Omega$ such that $u_{n_{0}+k}\left(x_{0}\right)-u_{n_{0}+k-1}\left(x_{0}\right) \geq M-\delta$. Now we apply Lemma 4.3 for $\lambda_{1}=M-\delta, \lambda_{2}=M+\delta, \lambda_{3}=\delta$ and $\lambda_{4}=\delta$ and we get

$$
\begin{aligned}
u_{n_{0}+k-1}\left(x_{0}\right), u_{n_{0}+k-1}\left(x_{1}\right) & \geq u_{n_{0}+k-2}\left(x_{1}\right)+\frac{2(M-\delta)}{\alpha}-(M+\delta)-\frac{2(1-\alpha)}{\alpha}-\delta \\
& =M\left(\frac{2}{\alpha}-1\right)-\delta \frac{4}{\alpha} \geq M-\delta \frac{2}{\alpha} .
\end{aligned}
$$

If we repeat the argument for $x_{1}$, but now with $\lambda_{1}=M-\delta \frac{2}{\alpha}$, we obtain

$$
u_{n_{0}+k-2}\left(x_{1}\right), u_{n_{0}+k-2}\left(x_{2}\right) \geq u_{n_{0}+k-3}\left(x_{2}\right)+M-\delta\left(\left(\frac{2}{\alpha}\right)^{2}+\frac{2}{\alpha}\right) .
$$

Inductively, we obtain a sequence $x_{l}, 1 \leq l \leq k-1$ such that

$$
u_{n_{0}+k-l}\left(x_{l-1}\right), u_{n_{0}+k-l}\left(x_{l}\right) \geq u_{n_{0}+k-l-1}\left(x_{l}\right)+M-\delta \sum_{t=1}^{l}\left(\frac{2}{\alpha}\right)^{t} .
$$

In Lemma 4.3 we require $\lambda_{3}<\lambda_{1}$, so we need $k(\delta)$ to satisfy

$$
M-\delta \sum_{t=1}^{l}\left(\frac{2}{\alpha}\right)^{t}>\delta
$$

that is,

$$
M>\delta \sum_{t=0}^{l}\left(\frac{2}{\alpha}\right)^{t}
$$

for $1 \leq l \leq k-1$, as the right hand side term grows with $l$, it is enough to check it for $l=k-1$. Since

$$
\sum_{t=1}^{l}\left(\frac{2}{\alpha}\right)^{t}=\frac{2}{\alpha} \frac{\left(\frac{2}{\alpha}\right)^{l}-1}{\frac{2}{\alpha}-1} \leq\left(\frac{2}{\alpha}\right)^{l+1}-1 \leq\left(\frac{2}{\alpha}\right)^{l+1},
$$

we obtain

$$
u_{n_{0}+k-l}\left(x_{l-1}\right) \geq u_{n_{0}+k-l-1}\left(x_{l}\right)+M-\delta\left(\frac{2}{\alpha}\right)^{l+1} .
$$


Adding this inequalities for $1 \leq l \leq k-1$, and $u_{n_{0}+k}\left(x_{0}\right)-u_{n_{0}+k-1}\left(x_{0}\right) \geq M-\delta$ we get

$$
u_{n_{0}+k}\left(x_{0}\right) \geq u_{n_{0}}\left(x_{k-1}\right)+k M-\delta \sum_{l=0}^{k-1}\left(\frac{2}{\alpha}\right)^{l+1} .
$$

From the last inequality and the condition for $k(\delta)$, since

$$
\sum_{l=0}^{k-1}\left(\frac{2}{\alpha}\right)^{l+1}=\sum_{l=1}^{k}\left(\frac{2}{\alpha}\right)^{l} \leq\left(\frac{2}{\alpha}\right)^{k+1},
$$

we have

$$
u_{n_{0}+k}\left(x_{0}\right) \geq u_{n_{0}}\left(x_{k-1}\right)+k M-\delta\left(\frac{2}{\alpha}\right)^{k+1}
$$

for all $k$ such that $M>\delta\left(\frac{2}{\alpha}\right)^{k+1}$. For $k+1=\left\lfloor\frac{\log \frac{M}{\delta}}{\log \frac{2}{\alpha}}\right\rfloor$ this gives

$$
u_{n_{0}+k}\left(x_{0}\right) \geq u_{n_{0}}\left(x_{k-1}\right)+\left(\frac{\log \frac{M}{\delta}}{\log \frac{2}{\alpha}}-3\right) M
$$

which is a contradiction since

$$
\lim _{\delta \rightarrow 0^{+}} \frac{\log \frac{M}{\delta}}{\log \frac{2}{\alpha}}=\infty
$$

and the sequence $u_{n}$ is bounded. We have that $u_{n} \rightarrow u$ uniformly, therefore the result follows by passing to the limit in the recursive definition of $u_{n}$. In fact, that the uniform limit of the sequence $u_{n}$ is a solution to the DPP is immediate since from the uniform convergence we can pass to the limit as $n \rightarrow \infty$ in all the terms of the DPP formula.

Now we want to prove that this solution to the DPP, $u$, is unique and that it gives the value of the game. To this end we have to take special care of the fact that the game ends (or not) almost surely. First, we deal with the case $\beta_{1}, \beta_{2}>0$, $\sup _{\Omega} f<0$ or $\inf _{\Omega} f>0$. We apply a martingales argument to handle this cases. In other cases we also use the construction of the sequence $u_{k}$.

Lemma 4.5. Assume that $\beta_{1}, \beta_{2}>0, \sup f<0$ or $\inf f>0$. Then, if $v$ is a $p_{1}-p_{2}$-harmonious function for $g_{v}$ and $f_{v}$ such that $g_{v} \leq g_{u_{I}}$ and $f_{v} \leq f_{u_{I}}$, then $v \leq u_{I}$

Proof. By choosing a strategy according to the points where the maximal values of $v$ are attained, we show that Player I can obtain that a certain process is a submartingale. The optional stopping theorem then implies that the expectation of the process under this strategy is bounded by $v$. Moreover, this process provides an lower bound for $u_{\mathrm{I}}$. 
Player II follows any strategy and Player I follows a strategy $S_{\mathrm{I}}^{0}$ such that at $x_{k-1} \in \Omega$ he chooses $\Gamma$ as follows:

$$
\begin{aligned}
\gamma=1 \text { if } \frac{\alpha_{1}}{2}\left\{\sup _{y \in B_{\varepsilon}(x)} u(y)+\inf _{y \in B_{\varepsilon}(x)} u(y)\right\}+\beta_{1} f_{B_{\varepsilon}(x)} u(y) d y \\
>\frac{\alpha_{2}}{2}\left\{\sup _{y \in B_{\varepsilon}(x)} u(y)+\inf _{y \in B_{\varepsilon}(x)} u(y)\right\}+\beta_{2} f_{B_{\varepsilon}(x)} u(y) d y
\end{aligned}
$$

and $\gamma=0$ othewise,

and to step to a point that almost maximize $v$, that is, to a point $x_{k} \in B_{\varepsilon}\left(x_{k-1}\right)$ such that

$$
v\left(x_{k}\right) \geq \sup _{B_{\varepsilon}\left(x_{k-1}\right)} v-\eta 2^{-k}
$$

for some fixed $\eta>0$. We start from the point $x_{0}$. It follows that

$$
\begin{aligned}
& \mathbb{E}_{S_{\mathrm{I}}, S_{\mathrm{II}}^{0}}^{x_{0}}\left[v\left(x_{k}\right)-\eta 2^{-k} \mid x_{0}, \ldots, x_{k-1}\right] \\
& \geq \max _{i \in\{1,2\}}\left(\frac{\alpha_{i}}{2}\left\{\inf _{B_{\varepsilon}\left(x_{k-1}\right)} v-\eta 2^{-k}+\sup _{B_{\varepsilon}\left(x_{k-1}\right)} v\right\}+\beta_{i} f_{B_{\varepsilon}\left(x_{k-1}\right)} v d y\right)-\eta 2^{-k} \\
& \geq v\left(x_{k-1}\right)-\varepsilon^{2} f\left(x_{k-1}\right)-\eta 2^{-(k-1)},
\end{aligned}
$$

where we have estimated the strategy of Player II inf and used the fact that $v$ is $p_{1}-p_{2}$-harmonious. Thus

$$
M_{k}=v\left(x_{k}\right)+\varepsilon^{2} \sum_{n=0}^{k} f\left(x_{n}\right)-\eta 2^{-k}
$$

is a submartingale.

Now we observe the following: if $\beta_{1}, \beta_{2}>0$ then the game ends almost surely and we can continue (see below). If $\sup f<0$ we have that the fact that $M_{k}$ is a submartingale implies that the game ends in a finite number of moves (that can be estimated). In the case inf $f>0$ if the game does not end in a finite number of moves then we have to play until the accumulated payoff (recall that $f$ gives the running payoff) is greater than $v$ and then choose a strategy that ends the game almost surely (for example pointing to some prescribed point $x_{0}$ outside $\Omega$ ).

Since $g_{v} \leq g_{u_{\mathrm{I}}}$ and $f_{v} \leq f_{u_{\mathrm{I}}}$, we deduce

$$
\begin{aligned}
u_{\mathrm{I}}\left(x_{0}\right) & =\sup _{S_{\mathrm{I}}} \inf _{S_{\mathrm{II}}} \mathbb{E}_{S_{\mathrm{I}}, S_{\mathrm{II}}}^{x_{0}}\left[g_{u_{\mathrm{I}}^{\varepsilon}}\left(x_{\tau}\right)+\varepsilon^{2} \sum_{n=0}^{\tau} f\left(x_{n}\right)\right] \\
& \geq \inf _{S_{\mathrm{II}}} \mathbb{E}_{S_{\mathrm{I}}^{0}, S_{\mathrm{II}}}^{x_{0}}\left[g_{v}\left(x_{\tau}\right)+\varepsilon^{2} \sum_{n=0}^{\tau} f\left(x_{n}\right)-\eta 2^{-\tau}\right] \\
& \geq \inf _{S_{\mathrm{II}}} \liminf _{k \rightarrow \infty} \mathbb{E}_{S_{\mathrm{I}}^{0}, S_{\mathrm{II}}}^{x_{0}}\left[v\left(x_{\tau \wedge k}\right)+\varepsilon^{2} \sum_{n=0}^{\tau \wedge k} f\left(x_{n}\right)-\eta 2^{-(\tau \wedge k)}\right] \\
& \geq \inf _{S_{\mathrm{II}}} \mathbb{E}_{S_{\mathrm{I}}^{0}, S_{\mathrm{II}}}\left[M_{0}\right]=v\left(x_{0}\right)-\eta,
\end{aligned}
$$

where $\tau \wedge k=\min (\tau, k)$, and we used Fatou's lemma as well as the optional stopping theorem for $M_{k}$. Since $\eta$ is arbitrary this proves the claim. 
A symmetric result can be proved for $u_{\mathrm{II}}$, hence we obtain the following result:

Theorem 4.6. Assume that $\beta_{1}, \beta_{2}>0, \sup f<0$ or $\inf f>0$. Then there exists a unique $p_{1}-p_{2}$-harmonious function. Even more the game has a value, that is $u_{I}=u_{I I}$, which coincides with the unique $p_{1}$ - $p_{2}$-harmonious function.

Proof. Let $u$ be a $p_{1}-p_{2}$-harmonious function, that we know that exits by Lemma 4.2. From the definition of the game values we know that $u_{I} \leq u_{I I}$. Then by Lemma 4.5 we have that

$$
u_{I} \leq u_{I I} \leq u \leq u_{I} .
$$

This is $u_{I}=u_{I I}=u$. Since we can repeat the argument for any $p_{1}$ - $p_{2}$-harmonious function, uniqueness follows.

Remark 4.7. Note that if we have a sub- $p_{1}-p_{2}$-harmonious function $u$, then $v$ given by $v=u-C$ in $\Omega$ and $v=u$ in $\Gamma_{\varepsilon}$ is sub- $p_{1}-p_{2}$-harmonious for every constant $C>0$. In this way we can obtain a sub- $p_{1}-p_{2}$-harmonious function smaller that any super- $p_{1}-p_{2}$-harmonious function, and then if we start the above construction with this function we get the smallest $p_{1}-p_{2}$-harmonious function. That is, there exists a minimal $p_{1}-p_{2}$-harmonious function. We can do the analogous construction to get the larger $p_{1}-p_{2}$-harmonious function (the maximal $p_{1}-p_{2}$-harmonious function).

We now tackle the remaining case in which $f \equiv 0$ and one of the $\beta_{i}$ is zero (that is the same as saying that one of the $\alpha_{i}$ is equal to one).

Theorem 4.8. There exists a unique $p_{1}$ - $p_{2}$-harmonious function when $\alpha_{1}=1$, $\alpha_{2}>0$ and $f \equiv 0$.

Proof. Supposed not, this is, we have $u, v$, such that

$$
\begin{aligned}
& v(x)=\max \left\{\frac{1}{2}\left(\sup _{B_{\varepsilon}(x)} v+\inf _{B_{\varepsilon}(x)} v\right), \frac{\alpha}{2}\left(\sup _{B_{\varepsilon}(x)} v+\inf _{B_{\varepsilon}(x)} v\right)+\beta f_{B_{\varepsilon}(x)} v\right\} \\
& u(x)=\max \left\{\frac{1}{2}\left(\sup _{B_{\varepsilon}(x)} u+\inf _{B_{\varepsilon}(x)} u\right), \frac{\alpha}{2}\left(\sup _{B_{\varepsilon}(x)} u+\inf _{B_{\varepsilon}(x)} u\right)+\beta f_{B_{\varepsilon}(x)} u\right\}
\end{aligned}
$$

in $\Omega$ and

$$
u=v=g
$$

on $\Gamma_{\varepsilon}$ with

$$
\|u-v\|_{\infty}=M>0 .
$$

As we observed in Remark 4.7 we can assume $u \geq v$ (just take $v$ the minimal solution to the DPP). Now we want to build a point where the difference between $u$ and $v$ is almost attained and $v$ has a large variation in the ball of radius $\varepsilon$ around this point (all this has to be carefully quantified). First, we apply a compactness argument. We know that

$$
\bar{\Omega}_{\frac{\varepsilon}{4}} \subset \bigcup_{x \in \Omega} B_{\frac{\varepsilon}{2}}(x) .
$$

As $\bar{\Omega}_{\frac{\varepsilon}{4}}$ is compact there exists $y_{i}$ such that

$$
\bar{\Omega}_{\frac{\varepsilon}{4}} \subset \bigcup_{i=1}^{k} B_{\frac{\bar{\varepsilon}}{2}}\left(y_{i}\right) .
$$


Let $A=\left\{i \in\{1, \ldots, k\}: u\right.$ or $v$ are not constant on $\left.B_{\frac{\varepsilon}{2}}\left(y_{i}\right)\right\}$ and let $\lambda>0$ such that for every $i \in A$

$$
\sup _{B_{\varepsilon}\left(y_{i}\right)} u-\inf _{B_{\varepsilon}\left(y_{i}\right)} u>\left(4+\frac{4 \beta}{\alpha}\right) \lambda \text { or } \sup _{B_{\varepsilon}\left(y_{i}\right)} v-\inf _{B_{\varepsilon}\left(y_{i}\right)} v>2 \lambda .
$$

We fix this $\lambda$. Now, for every $\delta$ such that $M, \lambda>\delta>0$, let $z \in \Omega$ such that $M-\delta<u(z)-v(z)$. Let

$$
O=\{x \in \Omega: u(x)=u(z) \text { and } v(x)=v(z)\} \subset \Omega .
$$

Take $\bar{z} \in \partial O \subset \bar{\Omega}$. Let $i_{0}$ such that $\bar{z} \in B_{\frac{\varepsilon}{2}}\left(y_{i_{0}}\right)$, we have

$$
B_{\frac{\varepsilon}{2}}\left(y_{i_{0}}\right) \cap O \neq \emptyset \quad \text { and } \quad B_{\frac{\varepsilon}{2}}\left(y_{i_{0}}\right) \cap O^{c} \neq \emptyset
$$

hence $i_{0} \in A$. Let $x_{0} \in B_{\frac{\varepsilon}{2}}\left(y_{i_{0}}\right) \cap O$. In this way we have obtained $x_{0}$ such that $u\left(x_{0}\right)-v\left(x_{0}\right)>M-\delta$ and one of the following holds:

(1)

$$
\sup _{B_{\varepsilon}\left(x_{0}\right)} u-\inf _{B_{\varepsilon}\left(x_{0}\right)} u>\left(4+\frac{4 \beta}{\alpha}\right) \lambda
$$

$$
\sup _{B_{\varepsilon}\left(x_{0}\right)} v-\inf _{B_{\varepsilon}\left(x_{0}\right)} v>2 \lambda .
$$

Let us show that in fact the second statement must hold. Supposed not, then the first holds and we have

$$
\sup _{B_{\varepsilon}\left(x_{0}\right)} v-\inf _{B_{\varepsilon}\left(x_{0}\right)} v \leq 2 \lambda
$$

Given that

$$
v\left(x_{0}\right) \geq \frac{1}{2}\left(\sup _{B_{\varepsilon}\left(x_{0}\right)} v+\inf _{B_{\varepsilon}\left(x_{0}\right)} v\right)
$$

we get

$$
v\left(x_{0}\right)+\lambda \geq \sup _{B_{\varepsilon}\left(x_{0}\right)} v
$$

Hence

$$
v\left(x_{0}\right)+\lambda+M \geq \sup _{B_{\varepsilon}\left(x_{0}\right)} v+M \geq \sup _{B_{\varepsilon}\left(x_{0}\right)} u .
$$

But we have more, since

$$
u\left(x_{0}\right)-v\left(x_{0}\right)>M-\delta>M-\lambda,
$$

we get

$$
u\left(x_{0}\right)+2 \lambda>\sup _{B_{\varepsilon}\left(x_{0}\right)} u,
$$

and

$$
\sup _{B_{\varepsilon}\left(x_{0}\right)} u>\inf _{B_{\varepsilon}\left(x_{0}\right)} u+\left(4+\frac{4 \beta}{\alpha}\right) \lambda
$$

Hence

$$
u\left(x_{0}\right)-\left(2+\frac{4 \beta}{\alpha}\right) \lambda>\inf _{B_{\varepsilon}\left(x_{0}\right)} u .
$$


If we bound the integral by the value of the supremum we can control all the terms in the DPP in terms of $u\left(x_{0}\right)$. We have

$$
\begin{aligned}
& u\left(x_{0}\right)= \max \left\{\frac{1}{2}\left(\sup _{B_{\varepsilon}\left(x_{0}\right)} u+\inf _{B_{\varepsilon}\left(x_{0}\right)} u\right), \frac{\alpha}{2}\left(\sup _{B_{\varepsilon}\left(x_{0}\right)} u+\inf _{B_{\varepsilon}\left(x_{0}\right)} u\right)+\beta f_{B_{\varepsilon}\left(x_{0}\right)} u\right\} \\
&< \max \left\{\frac{1}{2}\left(u\left(x_{0}\right)+2 \lambda+u\left(x_{0}\right)-\left(2+\frac{4 \beta}{\alpha}\right) \lambda\right),\right. \\
&\left.\frac{\alpha}{2}\left(u\left(x_{0}\right)+2 \lambda+u\left(x_{0}\right)-\left(2+\frac{4 \beta}{\alpha}\right) \lambda\right)+\beta\left(u\left(x_{0}\right)+2 \lambda\right)\right\} \\
&<\max \left\{u\left(x_{0}\right)-\frac{4 \beta}{\alpha} \lambda, u\left(x_{0}\right)\right\}=u\left(x_{0}\right),
\end{aligned}
$$

which is a contradiction. Hence we obtain that the second condition must hold, that is, we have

$$
\sup _{B_{\varepsilon}\left(x_{0}\right)} v-\inf _{B_{\varepsilon}\left(x_{0}\right)} v>2 \lambda
$$

Applying the DPP we get

$$
v\left(x_{0}\right) \geq \frac{1}{2}\left(\sup _{B_{\varepsilon}\left(x_{0}\right)} v+\inf _{B_{\varepsilon}\left(x_{0}\right)} v\right)
$$

together with the fact that

$$
\sup _{B_{\varepsilon}\left(x_{0}\right)} v-\inf _{B_{\varepsilon}\left(x_{0}\right)} v>2 \lambda
$$

then we conclude that

$$
v\left(x_{0}\right)>\inf _{B_{\varepsilon}\left(x_{0}\right)} v+\lambda .
$$

We have proved that there exists $x_{0}$ such that

$$
v\left(x_{0}\right)>\inf _{B_{\varepsilon}\left(x_{0}\right)} v+\lambda \quad \text { and } \quad u\left(x_{0}\right)-v\left(x_{0}\right)>M-\delta .
$$

Now we are going to build a sequence of points where the difference between $u$ and $v$ is almost maximal and where the value of $v$ decrease at least $\lambda$ in every step. Applying the DPP to $M-\delta<u\left(x_{0}\right)-v\left(x_{0}\right)$ and bounding the difference of the suprema by $M$ we get:

$$
M-\frac{2}{\alpha} \delta+\inf _{B_{\varepsilon}\left(x_{0}\right)} v<\inf _{B_{\varepsilon}\left(x_{0}\right)} u
$$

Let $x_{1}$ be such that $v\left(x_{0}\right)>v\left(x_{1}\right)+\lambda$ and $\inf _{B_{\varepsilon}\left(x_{0}\right)} v+\delta>v\left(x_{1}\right)$. We get

$$
M-\left(1+\frac{2}{\alpha}\right) \delta+v\left(x_{1}\right)<u\left(x_{1}\right) .
$$

To repeat this construction we need two things:

- In the last inequality if $\delta$ is small enough we have $u\left(x_{1}\right) \neq v\left(x_{1}\right)$, hence $x_{1} \in \Omega$.

- We know that $2 v\left(x_{1}\right) \geq \inf _{B_{\varepsilon}\left(x_{1}\right)} v+\sup _{B_{\varepsilon}\left(x_{1}\right)} v>v\left(x_{0}\right)+\inf _{B_{\varepsilon}\left(x_{1}\right)} v$. Hence, since $v\left(x_{0}\right)>v\left(x_{1}\right)+\lambda$, we get $v\left(x_{1}\right)>\inf _{B_{\varepsilon}\left(x_{1}\right)} v+\lambda$. 
Then we get

$$
v\left(x_{n-1}\right)>v\left(x_{n}\right)+\lambda
$$

and

$$
M-\left(\sum_{k=0}^{n}\left(\frac{2}{\alpha}\right)^{k}\right) \delta+v\left(x_{n}\right)<u\left(x_{n}\right) .
$$

We can repeat this argument as long as

$$
M-\left(\sum_{k=0}^{n}\left(\frac{2}{\alpha}\right)^{k}\right) \delta>0
$$

which is a contradiction with the fact that we know that $v$ is bounded.

Now we want to show that this unique function that satisfies the DPP is the game value. The key point of the proof is to construct an strategy based on the approximating sequence that we used to construct the solution.

Theorem 4.9. Given $f \equiv 0$ and $\alpha_{1}, \alpha_{2}>0$. The game has a value, that is $u_{I}=u_{I I}$, which coincides with the unique $p_{1}-p_{2}$-harmonious function.

Proof. Let $u$ be the unique $p_{1}-p_{2}$-harmonious function. We will show that $u \leq u_{I}$. The analogous result can be proved for $u_{I I}$ completing the proof.

Let us consider a function $u_{0}$, sub- $p_{1}-p_{2}$-harmonious smaller that $\inf _{\Omega} g$ at every point in $\Omega$. Starting with this $u_{0}$ we build the corresponding $u_{k}$ as in Lemma 4.4. We have that $u_{k} \rightarrow u$ as $k \rightarrow \infty$.

Now, given $\delta>0$ let $n>0$ be such that $u_{n}\left(x_{0}\right)>u\left(x_{0}\right)-\frac{\delta}{2}$. We build an strategy $S_{I}^{0}$ for Player I, in the firsts $n$ moves, given $x_{k-1}$ he will choose to move to a point that almost maximize $u_{n-k}$, that is, he chooses $x_{k} \in B_{\varepsilon}\left(x_{k-1}\right)$ such that

$$
u_{n-k}\left(x_{k}\right)>\sup _{B_{\varepsilon}\left(x_{k-1}\right)} u_{n-k}-\frac{\delta}{2 n} .
$$

and choose $\gamma$ in order to maximize

$$
\frac{\alpha_{i}}{2}\left\{\inf _{B_{\varepsilon}\left(x_{k-1}\right)} u_{n-k}-\frac{\delta}{2 n} \cdot+\sup _{B_{\varepsilon}\left(x_{k-1}\right)} u_{n-k}\right\}+\beta_{i} f_{B_{\varepsilon}\left(x_{k-1}\right)} u_{n-k} d y .
$$

After the first $n$ moves he will follow a strategy that ends the game almost surely (for example pointing in a fix direction).

We have

$$
\begin{aligned}
& \mathbb{E}_{S_{\mathrm{I}}^{0}, S_{\mathrm{II}}}^{x_{\mathrm{I}}}\left[u_{n-k}\left(x_{k}\right)+\frac{k \delta}{2 n} \mid x_{0}, \ldots, x_{k-1}\right] \\
& \geq \max _{i \in\{1,2\}}\left(\frac{\alpha_{i}}{2}\left\{\inf _{B_{\varepsilon}\left(x_{k-1}\right)} u_{n-k}-\frac{\delta}{2 n} \cdot+\sup _{B_{\varepsilon}\left(x_{k-1}\right)} u_{n-k}\right\}\right. \\
& \left.+\beta_{i} f_{B_{\varepsilon}\left(x_{k-1}\right)} u_{n-k} d y\right)-\frac{k \delta}{2 n} \\
& \geq u_{n-k+1}\left(x_{k-1}\right)+\frac{(k-1) \delta}{2 n},
\end{aligned}
$$


where we have estimated the strategy of Player II by inf and used the construction for the $u_{k}$ 's. Thus

$$
M_{k}= \begin{cases}u_{n-k}\left(x_{k}\right)+\frac{k \delta}{2 n}-\frac{\delta}{2} & \text { for } 0 \leq k \leq n, \\ M_{k}=\inf _{\Omega} g & \text { for } k>n,\end{cases}
$$

is a submartingale.

Now we have

$$
\begin{aligned}
u_{\mathrm{I}}\left(x_{0}\right) & =\sup _{S_{\mathrm{I}}} \inf _{S_{\mathrm{II}}} \mathbb{E}_{S_{\mathrm{I}}, S_{\mathrm{II}}}^{x_{0}}\left[g\left(x_{\tau}\right)\right] \\
& \geq \inf _{S_{\mathrm{II}}} \mathbb{E}_{S_{\mathrm{I}}^{0}, S_{\mathrm{II}}}^{x_{0}}\left[g\left(x_{\tau}\right)\right] \\
& \geq \inf _{S_{\mathrm{II}}} \liminf _{k \rightarrow \infty} \mathbb{E}_{S_{\mathrm{I}}^{0}, S_{\mathrm{II}}}^{x_{0}}\left[M_{k}\right] \\
& \geq \inf _{S_{\mathrm{II}}} \mathbb{E}_{S_{\mathrm{I}}^{0}, S_{\mathrm{II}}}\left[M_{0}\right]=u_{n}\left(x_{0}\right)-\frac{\delta}{2}>u\left(x_{0}\right)-\delta,
\end{aligned}
$$

where $\tau \wedge k=\min (\tau, k)$, and we used the optional stopping theorem for $M_{k}$. Since $\delta$ is arbitrary this proves the claim.

As an immediate corollary of our results in this section we obtain a comparison result for solutions to the DPP.

Corollary 4.10. If $v$ and $u$ are $p_{1}-p_{2}$-harmonious functions for $g_{v}, f_{v}$ and $g_{u}, f_{u}$, respectively such that $g_{v} \geq g_{u}$ and $f_{v} \geq f_{u}$, then $v \geq u$.

\section{Properties of harmonious functions AND CONVERGENCE}

First, we show some properties of $p_{1}$ - $p_{2}$-harmonious functions that we need to prove convergence as $\varepsilon \rightarrow 0$. We want to apply the following Arzela-Ascoli type lemma. For its proof see Lemma 4.2 from [30].

Lemma 5.1. Let $\left\{u_{\varepsilon}: \bar{\Omega} \rightarrow \mathbb{R}, \varepsilon>0\right\}$ be a set of functions such that

(1) there exists $C>0$ so that $\left|u_{\varepsilon}(x)\right|<C$ for every $\varepsilon>0$ and every $x \in \bar{\Omega}$,

(2) given $\eta>0$ there are constants $r_{0}$ and $\varepsilon_{0}$ such that for every $\varepsilon<\varepsilon_{0}$ and any $x, y \in \bar{\Omega}$ with $|x-y|<r_{0}$ it holds

$$
\left|u_{\varepsilon}(x)-u_{\varepsilon}(y)\right|<\eta \text {. }
$$

Then, there exists a uniformly continuous function $u: \bar{\Omega} \rightarrow \mathbb{R}$ and a subsequence still denoted by $\left\{u_{\varepsilon}\right\}$ such that

$$
u_{\varepsilon} \rightarrow u \quad \text { uniformly in } \bar{\Omega},
$$

as $\varepsilon \rightarrow 0$.

So our task now is to show that the family $u_{\varepsilon}$ satisfies the hypotheses of the previous lemma. To this end we need some bounds on the expected exit time in the case a player choose a certain strategy.

Let us start showing that $u_{\varepsilon}$ are uniformly bounded. In Lemma 4.2 we obtained a bound for the value of the game for a fixed $\varepsilon$, here we need a bound independent of $\varepsilon$. To this end, let us define what we understand by pulling in one direction: We fix a direction, that is, a unitary vector $v$ and at each turn of the game the Player strategy is given $S\left(x_{k-1}\right)=x_{k-1}+\left(\varepsilon-\varepsilon^{3} / 2^{k}\right) v$. 
Lemma 5.2. In a game where a player pulls in a fix direction the expectation of the exit time is bounded above by

$$
\mathbb{E}[\tau] \leq C \varepsilon^{-2}
$$

for some $C>0$ independent of $\varepsilon$.

Proof. First, let us assume without lost of generality that

$$
\Omega \subset\left\{x \in \mathbb{R}^{n}: 0<x_{n}<R\right\}
$$

and that the direction that the player is pulling to is $-e_{n}$. Then

$$
M_{k}=\left(x_{k}\right)_{n}+\frac{\varepsilon^{3}}{2^{k}}
$$

is a supermartingale. Indeed, if the random move occurs, then we know that the expectation of $\left(x_{k+1}\right)_{n}$ is equal to $\left(x_{k}\right)_{n}$. If the tug-of-war game is played we know that with probability one half $\left(x_{k+1}\right)_{n}=\left(x_{k}\right)_{n}-\varepsilon+\varepsilon^{3} / 2^{k}$ and if the other player moves $\left(x_{k+1}\right)_{n} \leq\left(x_{k}\right)_{n}+\varepsilon$, so the expectation is less or equal to $\left(x_{k}\right)_{n}+\frac{\varepsilon^{3}}{2^{k+1}}$.

Let us consider the expectation for $\left(M_{k+1}-M_{k}\right)^{2}$. If the random walk occurs, then the expectation is $\frac{\varepsilon^{2}}{n+2}+o\left(\varepsilon^{2}\right)$. Indeed,

$$
f_{B_{\varepsilon}} x_{n}^{2}=\frac{1}{n} f_{B_{\varepsilon}}|x|^{2}=\frac{1}{\varepsilon^{n} n\left|B_{1}\right|} \int_{0}^{\varepsilon} r^{2}\left|\partial B_{r}\right| d r=\frac{\left|\partial B_{1}\right|}{\varepsilon^{n} n\left|B_{1}\right|} \int_{0}^{\varepsilon} r^{n+1} d r=\frac{\varepsilon^{2}}{n+2} .
$$

If the tug-of-war occurs we know that with probability one half $\left(x_{k+1}\right)_{n}=\left(x_{k}\right)_{n}-$ $\varepsilon+\varepsilon^{3} / 2^{k}$, so the expectation is greater than or equal to $\frac{\varepsilon^{2}}{3}$.

Let us consider the expectation for $M_{k}^{2}-M_{k+1}^{2}$. We have,

$$
\mathbb{E}\left[M_{k}^{2}-M_{k+1}^{2}\right]=\mathbb{E}\left[\left(M_{k+1}-M_{k}\right)^{2}\right]+2 \mathbb{E}\left[\left(M_{k}-M_{k+1}\right) M_{k+1}\right] .
$$

As $\left(x_{k}\right)_{n}$ is positive, we have $2 \mathbb{E}\left[\left(M_{k}-M_{k+1}\right) M_{k+1}\right] \geq 0$. Then $\mathbb{E}\left[M_{k}^{2}-M_{k+1}^{2}\right] \geq$ $\frac{\varepsilon^{2}}{n+2}$, so $M_{k}^{2}+\frac{k \varepsilon^{2}}{n+2}$ is a supermartingale. According to the optional stopping theorem for supermartingales

$$
\mathbb{E}\left[M_{\tau \wedge k}^{2}+\frac{(\tau \wedge k) \varepsilon^{2}}{n+2}\right] \leq M_{0}^{2}
$$

We have

$$
\mathbb{E}[(\tau \wedge k)] \frac{\varepsilon^{2}}{n+2} \leq \mathcal{M}_{0}^{2}-E\left[M_{\tau \wedge k}^{2}\right] \leq M_{0}^{2} .
$$

Taking limit in $k$, we get a bound for the expected exit time,

$$
\mathbb{E}[\tau] \leq(n+2) M_{0}^{2} \varepsilon^{-2},
$$

so, the statement holds for $C=(n+2) R^{2}$.

Lemma 5.3. A $f$ - $p_{1}-p_{2}$-harmonious function $u_{\varepsilon}$ with boundary values $g$ satisfies

$$
\inf _{y \in \Gamma_{\varepsilon}} g(y)+C \inf _{y \in \Omega} f(y) \leq u_{\varepsilon}(x) \leq \sup _{y \in \Gamma_{\varepsilon}} g(y)+C \sup _{y \in \Omega} f(y) .
$$

Proof. We use the connection to games. Let one of the players choose a strategy of pulling in a fix direction. Then

$$
\mathbb{E}[\tau] \leq C \varepsilon^{-2}
$$


and this gives the upper bound

$$
\mathbb{E}\left[g\left(X_{\tau}\right)+\varepsilon^{2} \sum_{n=0}^{\tau} f\left(X_{n}\right)\right] \leq \sup _{y \in \Gamma_{\varepsilon}} g(y)+E[\tau] \varepsilon^{2} \sup _{y \in \Omega} f(y) \leq \sup _{y \in \Gamma_{\varepsilon}} g(y)+C \sup _{y \in \Omega} f(y) .
$$

The lower bound follows analogously.

Let us show now that the $u_{\varepsilon}$ are asymptotically uniformly continuous. First we need a lemma that bound the expectation for the exit time when one player is pulling towards a fix point.

Lemma 5.4. Let us consider an annular domain $B_{R}(y) \backslash \bar{B}_{\delta}(y)$ and a game where in each round an $\varepsilon$ step tug-of-war is played inside $B_{R}(y)$ or the token moves at random with uniform probability in $B_{R}(y) \cap B_{\varepsilon}(x)$. Assume that one of the players pulls towards $y$. Then

$$
\mathbb{E}^{x_{0}}\left(\tau^{*}\right) \leq \frac{C(R / \delta) \operatorname{dist}\left(\partial B_{\delta}(y), x_{0}\right)+o(1)}{\varepsilon^{2}},
$$

for $x_{0} \in B_{R}(y) \backslash \bar{B}_{\delta}(y)$. Here $o(1) \rightarrow 0$ as $\varepsilon \rightarrow 0$.

Proof. Let us denote

$$
h_{\varepsilon}(x)=\mathbb{E}^{x}(\tau)
$$

By symmetry we know that $h_{\varepsilon}$ is radial and it is easy to see that it is increasing in $r=|x-y|$. If we assume that the other player wants to maximize the expectation for the exit time and that the random move or tug-of-war is chosen in the same way, we have that the function $h_{\varepsilon}$ satisfies a dynamic programming principle

$$
h_{\varepsilon}(x)=\max \left\{\frac{1}{2}\left(\max _{B_{\varepsilon}(x) \cap B_{R}(y)} h_{\varepsilon}+\min _{B_{\varepsilon}(x) \cap B_{R}(y)} h_{\varepsilon}\right), f_{B_{\varepsilon}(x) \cap B_{R}(y)} h_{\varepsilon} d z\right\}+1
$$

by the above assumptions and that the number of steps always increases by one when making a step. Further, we denote $v_{\varepsilon}(x)=\varepsilon^{2} h_{\varepsilon}(x)$ and obtain

$$
v_{\varepsilon}(x)=\max \left\{\frac{1}{2}\left(\sup _{B_{\varepsilon}(x) \cap B_{R}(y)} v_{\varepsilon}+\inf _{B_{\varepsilon}(x) \cap B_{R}(y)} v_{\varepsilon}\right), f_{B_{\varepsilon}(x) \cap B_{R}(y)} v_{\varepsilon} d z\right\}+\varepsilon^{2}
$$

This induces us to look for a function $v$ such that

$$
\begin{aligned}
& v(x) \geq f_{B_{\varepsilon}(x)} v d z+\varepsilon^{2} \\
& \text { and } \\
& v(x) \geq \frac{1}{2}\left(\sup _{B_{\varepsilon}(x)} v+\inf _{B_{\varepsilon}(x)} v\right)+\varepsilon^{2} .
\end{aligned}
$$

Note that for small $\varepsilon$ this is a sort of discrete version to the following inequalities

$$
\begin{cases}\Delta v(x) \leq-2(n+2), & x \in B_{R+\varepsilon}(y) \backslash \bar{B}_{\delta-\varepsilon}(y), \\ \Delta_{\infty} v(x) \leq-2, & x \in B_{R+\varepsilon}(y) \backslash \bar{B}_{\delta-\varepsilon}(y) .\end{cases}
$$


This leads us to consider the problem

$$
\begin{cases}\Delta v(x)=-2(n+2), & x \in B_{R+\varepsilon}(y) \backslash \bar{B}_{\delta}(y), \\ v(x)=0, & x \in \partial B_{\delta}(y), \\ \frac{\partial v}{\partial \nu}=0, & x \in \partial B_{R+\varepsilon}(y),\end{cases}
$$

where $\frac{\partial u}{\partial \nu}$ refers to the normal derivative. The solution to this problem is radially symmetric and strictly increasing in $r=|x-y|$. It takes the form

$$
v(r)=-a r^{2}-b r^{2-N}+c,
$$

if $N>2$ and

$$
v(r)=-a r^{2}-b \log (r)+c,
$$

if $N=2$. If we extend this $v$ to $B_{\delta}(y) \backslash \bar{B}_{\delta-\varepsilon}(y)$, it satisfies $\Delta v(x)=-2(N+2)$ in $B_{R+\varepsilon}(y) \backslash \bar{B}_{\delta-\varepsilon}(y)$. We know that

$$
\Delta_{\infty} v=v_{r r} \leq v_{r r}+\frac{N-1}{r} v_{r}=\Delta v
$$

Thus, $v$ satisfy the inequalities (5.4). Then, the classical calculation shows that $v$ satisfies (5.3) for each $B_{\varepsilon}(x) \subset B_{R+\varepsilon}(y) \backslash \bar{B}_{\delta-\varepsilon}(y)$.

In addition, as $v$ is increasing in $r$, it holds for each $x \in B_{R}(y) \backslash \bar{B}_{\delta}(y)$ that

$$
f_{B_{\varepsilon}(x) \cap B_{R}(y)} v d z \leq f_{B_{\varepsilon}(x)} v d z \leq v(x)-\varepsilon^{2}
$$

and

$$
\frac{1}{2}\left(\sup _{B_{\varepsilon}(x) \cap B_{R}(y)} v_{+} \inf _{B_{\varepsilon}(x) \cap B_{R}(y)} v\right) \leq \frac{1}{2}\left(\sup _{B_{\varepsilon}(x)} v_{+} \inf _{B_{\varepsilon}(x)} v\right) \leq v(x)-\varepsilon^{2} .
$$

It follows that

$$
\begin{aligned}
& \mathbb{E}\left[v\left(x_{k}\right)+k \varepsilon^{2} \mid x_{0}, \ldots, x_{k-1}\right] \\
& \quad \leq \max \left\{\frac{1}{2}\left(\sup _{B_{\varepsilon}\left(x_{k-1}\right) \cap B_{R}(y)} v+\inf _{B_{\varepsilon}\left(x_{k-1}\right) \cap B_{R}(y)} v\right), f_{B_{\varepsilon}\left(x_{k-1}\right) \cap B_{R}(y)} v d z\right\} \\
& \quad \leq v\left(x_{k-1}\right)+(k-1) \varepsilon^{2},
\end{aligned}
$$

if $x_{k-1} \in B_{R}(y) \backslash \bar{B}_{\delta}(y)$. Thus $v\left(x_{k}\right)+k \varepsilon^{2}$ is a supermartingale, and the optional stopping theorem yields

$$
\mathbb{E}^{x_{0}}\left[v\left(x_{\tau^{*} \wedge k}\right)+\left(\tau^{*} \wedge k\right) \varepsilon^{2}\right] \leq v\left(x_{0}\right) .
$$

Because $x_{\tau^{*}} \in \bar{B}_{\delta}(y) \backslash \bar{B}_{\delta-\varepsilon}(y)$, we have

$$
0 \leq-\mathbb{E}^{x_{0}}\left[v\left(x_{\tau^{*}}\right)\right] \leq o(1)
$$

Furthermore, the estimate

$$
0 \leq v\left(x_{0}\right) \leq C(R / \delta) \operatorname{dist}\left(\partial B_{\delta}(y), x_{0}\right)
$$

holds for the solutions of (5.5). Thus, by passing to the limit as $k \rightarrow \infty$, we obtain

$$
\varepsilon^{2} \mathbb{E}^{x_{0}}\left[\tau^{*}\right] \leq v\left(x_{0}\right)-\mathbb{E}\left[u\left(x_{\tau^{*}}\right)\right] \leq C(R / \delta)\left(\operatorname{dist}\left(\partial B_{\delta}(y), x_{0}\right)+o(1)\right) .
$$

This completes the proof. 
Next we derive a uniform bound and estimate for the asymptotic continuity of the family of $p_{1}-p_{2}$-harmonious functions.

We assume here that $\Omega$ satisfies an exterior sphere condition: For each $y \in \partial \Omega$, there exists $B_{\delta}(z) \subset \mathbb{R}^{n} \backslash \Omega$ such that $y \in \partial B_{\delta}(z)$.

Lemma 5.5. Let $g$ be Lipschitz continuous in $\Gamma_{\varepsilon}$ and $f$ Lipschitz continuous in $\Omega$ such that $f \equiv 0$, inf $f>0$ or $\sup f<0$. The $p_{1}-p_{2}$-harmonious function $u_{\varepsilon}$ with data $g$ and $f$ satisfies

$$
\begin{aligned}
\left|u_{\varepsilon}(x)-u_{\varepsilon}(y)\right| \leq & \operatorname{Lip}(g)(|x-y|+\delta) \\
& +C(R / \delta)(|x-y|+o(1))\left(1+\|f\|_{\infty}\right)+\widetilde{C} \operatorname{Lip}(f)|x-y|,
\end{aligned}
$$

for every small enough $\delta>0$ and for every two points $x, y \in \Omega \cup \Gamma_{\varepsilon}$.

Proof. The case $x, y \in \Gamma_{\varepsilon}$ is clear. Thus, we can concentrate on the cases $x \in \Omega$ and $y \in \Gamma_{\varepsilon}$ as well as $x, y \in \Omega$.

We use the connection to games. Suppose first that $x \in \Omega$ and $y \in \Gamma_{\varepsilon}$. By the exterior sphere condition, there exists $B_{\delta}(z) \subset \mathbb{R}^{n} \backslash \Omega$ such that $y \in \partial B_{\delta}(z)$. Now Player I chooses a strategy of pulling towards $z$, denoted by $S_{I}^{z}$. Then

$$
M_{k}=\left|x_{k}-z\right|-C \varepsilon^{2} k
$$

is a supermartingale for a constant $C$ large enough independent of $\varepsilon$. Indeed,

$$
\begin{aligned}
& \mathbb{E}_{S_{\mathrm{I}}^{z}, S_{\mathrm{II}}}^{x_{0}}\left[\left|x_{k}-z\right| \mid x_{0}, \ldots, x_{k-1}\right] \\
& \leq \max _{i \in\{1,2\}}\left(\frac{\alpha_{i}}{2}\left\{\left|x_{k-1}-z\right|+\varepsilon-\varepsilon^{3}+\left|x_{k-1}-z\right|-\varepsilon\right\}+\beta_{i} f_{B_{\varepsilon}\left(x_{k-1}\right)}|x-z| d x\right) \\
& \quad \leq\left|x_{k-1}-z\right|+C \varepsilon^{2} .
\end{aligned}
$$

The first inequality follows from the choice of the strategy, and the second from the estimate

$$
f_{B_{\varepsilon}\left(x_{k-1}\right)}|x-z| d x \leq\left|x_{k-1}-z\right|+C \varepsilon^{2} .
$$

By the optional stopping theorem, this implies that

$$
\mathbb{E}_{S_{\mathrm{I}}^{z}, S_{\mathrm{II}}}^{x_{0}}\left[\left|x_{\tau}-z\right|\right] \leq\left|x_{0}-z\right|+C \varepsilon^{2} \mathbb{E}_{S_{\mathrm{I}}^{z}, S_{\mathrm{II}}}^{x_{0}}[\tau]
$$

Next we can estimate $\mathbb{E}_{S_{\mathrm{I}}^{z}, S_{\mathrm{II}}}^{x_{0}}[\tau]$ by the stopping time of Lemma 5.4. Let $R>0$ be such that $\Omega \subset B_{R}(z)$. Thus, by (5.2),

$$
\varepsilon^{2} \mathbb{E}_{S_{\mathrm{I}}^{z}, S_{\mathrm{II}}}^{x_{0}}[\tau] \leq \varepsilon^{2} \mathbb{E}_{S_{\mathrm{I}}^{z}, S_{\mathrm{II}}}^{x_{0}}\left[\tau^{*}\right] \leq C(R / \delta)\left(\operatorname{dist}\left(\partial B_{\delta}(z), x_{0}\right)+o(1)\right) .
$$

Since $y \in \partial B_{\delta}(z)$,

$$
\operatorname{dist}\left(\partial B_{\delta}(z), x_{0}\right) \leq\left|y-x_{0}\right|
$$

and thus, (5.8) implies

$$
\mathbb{E}_{S_{\mathrm{I}}^{z}, S_{\mathrm{II}}}^{x_{0}}\left[\left|x_{\tau}-z\right|\right] \leq C(R / \delta)\left(\left|x_{0}-y\right|+o(1)\right) .
$$

We get

$$
g(z)-C(R / \delta)(|x-y|+o(1)) \leq \mathbb{E}_{S_{\mathrm{I}}^{z}, S_{\mathrm{II}}}^{x_{0}}\left[g\left(x_{\tau}\right)\right]
$$


Thus, we obtain

$$
\begin{aligned}
\sup _{S_{\mathrm{I}}} \inf _{S_{\mathrm{II}}} & \mathbb{E}_{S_{\mathrm{I}}, S_{\mathrm{II}}}^{x_{0}}\left[g\left(x_{\tau}\right)+\varepsilon^{2} \sum_{n=0}^{\tau-1} f\left(x_{n}\right)\right] \\
& \geq \inf _{S_{\mathrm{II}}} \mathbb{E}_{S_{\mathrm{I}}^{z}, S_{\mathrm{II}}}^{x_{0}}\left[g\left(x_{\tau}\right)+\varepsilon^{2} \sum_{n=0}^{\tau-1} f\left(x_{n}\right)\right] \\
& \geq g(z)-C(R / \delta)\left(\left|x_{0}-y\right|+o(1)\right)-\varepsilon^{2} \inf _{S_{\mathrm{II}}} \mathbb{E}_{S_{\mathrm{I}}^{z}, S_{\mathrm{II}}}^{x_{0}}[\tau]\|f\|_{\infty} \\
& \geq g(y)-\operatorname{Lip}(g) \delta-C(R / \delta)\left(\left|x_{0}-y\right|+o(1)\right)\left(1+\|f\|_{\infty}\right) .
\end{aligned}
$$

The upper bound can be obtained by choosing for Player II a strategy where he points to $z$, and thus, (5.7) follows.

Finally, let $x, y \in \Omega$ and fix the strategies $S_{\mathrm{I}}, S_{\mathrm{II}}$ for the game starting at $x$. We define a virtual game starting at $y$ : we use the same coin tosses and random steps as the usual game starting at $x$. Furthermore, the players adopt their strategies $S_{\mathrm{I}}^{v}, S_{\mathrm{II}}^{v}$ from the game starting at $x$, that is, when the game position is $y_{k-1}$ a player chooses the step that would be taken at $x_{k-1}$ in the game starting at $x$. We proceed in this way until for the first time $x_{k} \in \Gamma_{\varepsilon}$ or $y_{k} \in \Gamma_{\varepsilon}$. At that point we have $\left|x_{k}-y_{k}\right|=|x-y|$, and we may apply the previous steps that work for $x_{k} \in \Omega$, $y_{k} \in \Gamma_{\varepsilon}$ or for $x_{k}, y_{k} \in \Gamma_{\varepsilon}$.

If we are in the case $f \equiv 0$ we are done. In the case $\inf _{y \in \Omega}|f(y)|>0$, as we know that the $u_{\varepsilon}$ are uniformly bounded according to Lemma 5.3, we have that the expected exit time is bounded by

$$
\widetilde{C}=\frac{\max _{y \in \Gamma_{\varepsilon}}|g(y)|+C \max _{y \in \Omega}|f(y)|}{\inf _{y \in \Omega}|f(y)|} .
$$

So the expected difference in the running payoff in the game starting at $x$ and the virtual one is bounded by $\widetilde{C} \operatorname{Lip}(f)|x-y|$, because $\left|x_{i}-y_{i}\right|=|x-y|$ for all $0 \leq i \leq k$.

Corollary 5.6. Let $\left\{u_{\varepsilon}\right\}$ be a family of $p_{1}-p_{2}$-harmonious. Then there exists a uniformly continuous $u$ and a subsequence still denoted by $\left\{u_{\varepsilon}\right\}$ such that

$$
u_{\varepsilon} \rightarrow u \quad \text { uniformly in } \bar{\Omega} \text {. }
$$

Proof. Using Lemmas 5.3 and 5.5 we get that the family $u_{\varepsilon}$ satisfies the hypothesis of the compactness Lemma 5.1.

Theorem 5.7. Any uniform limit of a subsequence of $u_{\varepsilon}$, $u$, (that there is such was subsequence was proved in Corollary 5.6) is a viscosity solution to (1.2) taking $f / 2$ as the running pay-off function.

Proof. First, we observe that $u=g$ on $\partial \Omega$ due to $u_{\varepsilon}=g$ on $\partial \Omega$ for all $\varepsilon>0$. Hence, we can focus our attention on showing that $u$ is $p_{1}$ - $p_{2}$-harmonic inside $\Omega$ in the viscosity sense. To this end, we recall from [28] an estimate that involves the regular Laplacian $(p=2)$ and an approximation for the infinity Laplacian $(p=\infty)$. Choose a point $x \in \Omega$ and a $C^{2}$-function $\phi$ defined in a neighborhood of $x$. Note that since $\phi$ is continuous then we have

$$
\min _{y \in \bar{B}_{\varepsilon}(x)} \phi(y)=\inf _{y \in B_{\varepsilon}(x)} \phi(y)
$$


for all $x \in \Omega$. Let $x_{1}^{\varepsilon}$ be the point at which $\phi$ attains its minimum in $\bar{B}_{\varepsilon}(x)$

$$
\phi\left(x_{1}^{\varepsilon}\right)=\min _{y \in \bar{B}_{\varepsilon}(x)} \phi(y) .
$$

It follows from the Taylor expansions in [28] that

$$
\begin{aligned}
& \frac{\alpha}{2}\left(\max _{y \in \bar{B}_{\varepsilon}(x)} \phi(y)+\min _{y \in \bar{B}_{\varepsilon}(x)} \phi(y)\right)+\beta f_{B_{\varepsilon}(x)} \phi(y) d y-\phi(x) \\
& \geq \frac{\varepsilon^{2}}{2(n+p)}\left\{(p-2)\left\langle D^{2} \phi(x)\left(\frac{x_{1}^{\varepsilon}-x}{\varepsilon}\right),\left(\frac{x_{1}^{\varepsilon}-x}{\varepsilon}\right)\right\rangle+\Delta \phi(x)\right\} \\
& \quad+o\left(\varepsilon^{2}\right) .
\end{aligned}
$$

Suppose that $\phi$ touches $u$ at $x$ strictly from below. We want to prove that $F^{*}\left(\nabla \phi(x), D^{2} \phi(x)\right) \geq f(x)$. By the uniform convergence, there exists sequence $\left\{x_{\varepsilon}\right\}$ converging to $x$ such that $u_{\varepsilon}-\phi$ has an approximate minimum at $x_{\varepsilon}$, that is, for $\eta_{\varepsilon}>0$, there exists $x_{\varepsilon}$ such that

$$
u_{\varepsilon}(x)-\phi(x) \geq u_{\varepsilon}\left(x_{\varepsilon}\right)-\phi\left(x_{\varepsilon}\right)-\eta_{\varepsilon} .
$$

Moreover, considering $\tilde{\phi}=\phi-u_{\varepsilon}\left(x_{\varepsilon}\right)-\phi\left(x_{\varepsilon}\right)$, we can assume that $\phi\left(x_{\varepsilon}\right)=u_{\varepsilon}\left(x_{\varepsilon}\right)$. Thus, by recalling the fact that $u_{\varepsilon}$ is $p_{1}-p_{2}$-harmonious, we obtain

$$
\eta_{\varepsilon} \geq \varepsilon^{2} \frac{f\left(x_{\varepsilon}\right)}{2}-\phi\left(x_{\varepsilon}\right)+\max _{i \in\{1,2\}}\left\{\frac{\alpha_{i}}{2}\left(\max _{\bar{B}_{\varepsilon}\left(x_{\varepsilon}\right)} \phi+\min _{\bar{B}_{\varepsilon}\left(x_{\varepsilon}\right)} \phi\right)+\beta_{i} f_{B_{\varepsilon}\left(x_{\varepsilon}\right)} \phi(y) d y\right\},
$$

and thus, by (5.9), and choosing $\eta_{\varepsilon}=o\left(\varepsilon^{2}\right)$, we have

$$
\begin{aligned}
0 \geq \frac{\varepsilon^{2}}{2} \max _{i \in\{1,2\}}\left\{\alpha_{i}\left\langle D^{2} \phi\left(x_{\varepsilon}\right)\left(\frac{x_{1}^{\varepsilon}-x_{\varepsilon}}{\varepsilon}\right),\left(\frac{x_{1}^{\varepsilon}-x_{\varepsilon}}{\varepsilon}\right)\right\rangle+\theta_{i} \Delta \phi\left(x_{\varepsilon}\right)\right\} \\
+\varepsilon^{2} \frac{f\left(x_{\varepsilon}\right)}{2}+o\left(\varepsilon^{2}\right) .
\end{aligned}
$$

Next we need to observe that

$$
\left\langle D^{2} \phi\left(x_{\varepsilon}\right)\left(\frac{x_{1}^{\varepsilon}-x_{\varepsilon}}{\varepsilon}\right),\left(\frac{x_{1}^{\varepsilon}-x_{\varepsilon}}{\varepsilon}\right)\right\rangle
$$

converge to $\Delta_{\infty} \phi(x)$ when $\nabla \phi(x) \neq 0$ and always is bounded in the limit by $\lambda_{\min }\left(D^{2} \phi(x)\right)$ and $\lambda_{\max }\left(D^{2} \phi(x)\right)$. Dividing by $\varepsilon^{2}$ and letting $\varepsilon \rightarrow 0$, we get

$$
F^{*}\left(\nabla \phi(x), D^{2} \phi(x)\right) \geq f(x) \text {. }
$$

Therefore $u$ is a viscosity supersolution.

To prove that $u$ is a viscosity subsolution, we use a reverse inequality to (5.9) by considering the maximum point of the test function and choose a smooth test function that touches $u$ from above.

Now, we just observe that this probabilistic approach provides an alternative existence proof of viscosity solutions to our PDE problem.

Corollary 5.8. Any limit function obtained as in Corollary 5.6 is a viscosity solution to the problem

$$
\begin{cases}\max \left\{-\Delta_{p_{1}} u,-\Delta_{p_{2}} u\right\}=f & \text { on } \Omega \\ u=g & \text { on } \partial \Omega .\end{cases}
$$

In particular, the problem has a solution. 
We proved that the problem has an unique solution using PDE methods, therefore we conclude that we have convergence as $\varepsilon \rightarrow 0$ of $u_{\varepsilon}$ (not only along subsequences).

Corollary 5.9. It holds that

$$
u_{\varepsilon} \rightarrow u \quad \text { uniformly in } \bar{\Omega},
$$

being $u$ the unique solution to the problem

$$
\begin{cases}\max \left\{-\Delta_{p_{1}} u,-\Delta_{p_{2}} u\right\}=f & \text { on } \Omega, \\ u=g & \text { on } \partial \Omega .\end{cases}
$$

\section{REFERENCES}

[1] T. Antunovic, Y. Peres, S. Sheffield, and S. Somersille. Tug-of-war and infinity Laplace equation with vanishing Neumann boundary condition. Comm. Partial Differential Equations, 37(10), 2012, 1839-1869.

[2] S.N. Armstrong and C.K. Smart. An easy proof of Jensen?s theorem on the uniqueness of infinity harmonic functions. Calc. Var. Partial Differential Equations, 37, (2010), 381-384.

[3] R. Atar and A. Budhiraja. A stochastic differential game for the inhomogeneous $\infty$-laplace equation. Ann. Probab., 38(2), (2010), 498-531.

[4] C. Bjorland, L.A. Caffarelli, and A. Figalli. Non-local gradient dependent operators. Adv. Math. 230(4-6), (2012), 1859-1894.

[5] C. Bjorland, L.A. Caffarelli, and A. Figalli. Nonlocal tug-of-war and the infinty fractional Laplacian. Comm. Pure Appl. Math. 65(3), (2012), 337-380.

[6] J. Busca, M. J. Esteban and A. Quaas. Nonlinear eigenvalues and bifurcation problems for Pucci's operators. Ann. Inst. H. Poincar Anal. Non Linaire, 22(2), (2005), 187-206.

[7] L. A. Caffarelli and X. Cabre, Fully nonlinear elliptic equations. Amer. Math. Soc. Colloquium Publications, 43. Providence, RI, 1995.

[8] F. Charro, J. Garcia Azorero and J. D. Rossi. A mixed problem for the infinity laplacian via Tug-of-War games. Calc. Var. Partial Differential Equations, 34(3), (2009), 307-320.

[9] M. G. Crandall, H. Ishii and P.-L. Lions. User's guide to viscosity solutions of second order partial differential equations. Bull. Amer. Math. Soc., 27, (1992), 1-67.

[10] P. Felmer, A. Quaas and M. Tang. On uniqueness for nonlinear elliptic equation involving the Pucci's extremal operator. J. Differential Equations 226(1), (2006), 80-98.

[11] E. Le Gruyer, On absolutely minimizing Lipschitz extensions and PDE $\Delta_{\infty}(u)=0$. NoDEA Nonlinear Differential Equations Appl. 14: 29-55, 2007.

[12] E. Le Gruyer and J. C. Archer, Harmonious extensions. SIAM J. Math. Anal. 29:279-292, 1998.

[13] D. Hartenstine and M. Rudd. Statistical functional equations and $p$ - harmonious functions. Adv. Nonlinear Stud. 13(1), (2013), 191-207.

[14] V. Julin and P. Juutinen. A new proof for the equivalence of weak and viscosity solutions for the p-Laplace equation. Comm. Partial Differential Equations, 37(5), (2012), 934-946.

[15] P. Juutinen, P. Lindqvist, and J. J. Manfredi. On the equivalence of viscosity solutions and weak solutions for a quasi-linear equation. SIAM J. Math. Anal., 33(3), (2001), 699-717.

[16] B. Kawohl, J.J. Manfredi, and M. Parviainen. Solutions of nonlinear PDEs in the sense of averages. J. Math. Pures Appl. 97(3), (2012), 173-188.

[17] S. Koike and K. Takahiro. Remarks on the comparison principle for quasilinear pde with no zeroth order terms. Commun. Pure Appl. Anal. 14(1), (2015), 133-142.

[18] P. Lindqvist and T. Lukkari, A curious equation involving the $\infty$-Laplacian, Adv. Calc. Var., 3, (2010), 409-421.

[19] Q. Liu and A. Schikorra. General existence of solutions to dynamic programming principle. Commun. Pure Appl. Anal. 14(1), (2015), 167-184.

[20] Q. Liu and A. Schikorra. A Game-Tree approach to discrete infinity Laplacian with running costs. arXiv preprint arXiv:1305.7372 (2013).

[21] J. G. Llorente. Mean value properties and unique continuation. Comm. Pure Appl. Anal. 14:1, (2015), 185-199. 
[22] J. G. Llorente, A note on unique continuation for solutions of the $\infty$-mean value property. Ann. Acad. Scient. Fennicae, 39, (2014), 473-483.

[23] G. Lu and P. Wang. Inhomogeneous infinity Laplace equation. Adv. Math., 217, (2008), 1838-1868.

[24] H. Luiro and M. Parviainen. Regularity for nonlinear stochastic games. Preprint.

[25] H. Luiro, M. Parviainen, and E. Saksman. Harnack's inequality for p-harmonic functions via stochastic games. Comm. Partial Differential Equations, 38.11, (2013), 1985-2003

[26] A. P. Maitra and W. D. Sudderth, Borel stochastic games with lim sup payoff. Ann. Probab. 21(2):861-885, 1996.

[27] A. P. Maitra and W. D. Sudderth, Discrete gambling and stochastic games. Applications of Mathematics 32, Springer-Verlag, 1996.

[28] J. J. Manfredi, M. Parviainen and J. D. Rossi, An asymptotic mean value characterization for p-harmonic functions. Proc. Amer. Math. Soc., 138, (2010), 881-889.

[29] J. J. Manfredi, M. Parviainen and Julio D. Rossi. Dynamic programming principle for tugof-war games with noise. ESAIM, Control, Opt. Calc. Var., 18, (2012), 81-90.

[30] J. J. Manfredi, M. Parviainen and Julio D. Rossi. On the definition and properties of $p$ harmonious functions. Ann. Scuola Nor. Sup. Pisa, 11, (2012), 215-241.

[31] K. Nystrom and M. Parviainen. Tug-of-war, market manipulation and option pricing. To appear in Math. Finance.

[32] A. Oberman, A convergent difference scheme for the infinity Laplacian: construction of absolutely minimizing Lipschitz extensions. Math. Comp., 74, (2005), 1217-1230.

[33] Y. Peres, O. Schramm, S. Sheffield and D. Wilson, Tug-of-war and the infinity Laplacian. J. Amer. Math. Soc., 22, (2009), 167-210.

[34] Y. Peres, S. Sheffield, Tug-of-war with noise: a game theoretic view of the p-Laplacian, Duke Math. J., 145(1), (2008), 91-120.

[35] A. Quaas and B. Sirakov, Existence results for nonproper elliptic equations involving the Pucci operator. Comm. Partial Differential Equations, 31, 7-9, (2006), 987-1003.

[36] E. Ruosteenoja. Local regularity results for value functions of tug-of-war with noise and running payoff. To appear in Adv. Calc. Var.

[37] S. R. S. Varadhan, Probability theory, Courant Lecture Notes in Mathematics 7, 2001.

Depto. Matemática, FCEyn, Buenos Aires University,

Ciudad Universitaria, Pab 1 (1428),

Buenos Aires, Argentina.

pblanc@dm.uba.ar, jpinasco@dm.uba.ar, jrossi@dm.uba.ar 